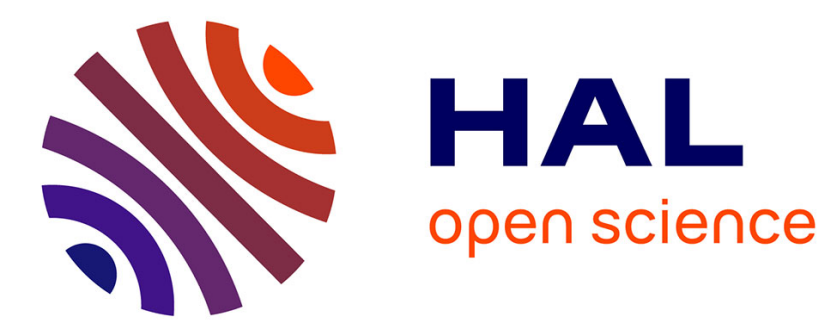

\title{
Simulation of three-dimensional nanoscale light interaction with spatially dispersive metals using a high order curvilinear DGTD method
}

Nikolai Schmitt, Claire Scheid, Jonathan Viquerat, Stéphane Lanteri

\section{- To cite this version:}

Nikolai Schmitt, Claire Scheid, Jonathan Viquerat, Stéphane Lanteri. Simulation of three-dimensional nanoscale light interaction with spatially dispersive metals using a high order curvilinear DGTD method. 2017. hal-01647074

\section{HAL Id: hal-01647074 \\ https://hal.science/hal-01647074}

Preprint submitted on 24 Nov 2017

HAL is a multi-disciplinary open access archive for the deposit and dissemination of scientific research documents, whether they are published or not. The documents may come from teaching and research institutions in France or abroad, or from public or private research centers.
L'archive ouverte pluridisciplinaire $\mathbf{H A L}$, est destinée au dépôt et à la diffusion de documents scientifiques de niveau recherche, publiés ou non, émanant des établissements d'enseignement et de recherche français ou étrangers, des laboratoires publics ou privés. 


\title{
Simulation of three-dimensional nanoscale light interaction with spatially dispersive metals using a high order curvilinear DGTD method
}

\author{
Nikolai Schmitt ${ }^{1}$, Claire Scheid ${ }^{2}$, Jonathan Viquerat ${ }^{1}$ and Stéphane Lanteri ${ }^{1}$ \\ ${ }^{1}$ Université Côte d'Azur, Inria, CNRS, LJAD, France \\ ${ }^{2}$ Université Côte d'Azur, LJAD, CNRS, Inria, France
}

\begin{abstract}
In this work, we present and study a flexible and accurate numerical solver in the context of three-dimensional computational nanophotonics. More precisely, we focus on the propagation of electromagnetic waves through metallic media described by a non-local dispersive model. For this model, we propose a discretization based on a high-order Discontinuous Galerkin timedomain method, along with a low-storage Runge-Kutta time scheme of order four. The semidiscrete stability of the scheme is analyzed for classical numerical fluxes, i.e. centered and upwind. Furthermore, the numerical treatment is enriched with an enhanced approximation of the geometry based on isoparametric curvilinear meshes. We finally assess our approach on several test cases, from academic to more physical ones.
\end{abstract}

Keywords: Nanoplasmonics, Maxwell's equations, Spatial dispersion, Nonlocal Drude model, Discontinuous Galerkin time-domain method, Isoparametric curvilinear elements

\section{Motivations and objectives}

\subsection{Physical context}

Nanophotonics [1] is where photonics merges with nanoscience and nanotechnology, and where spatial confinement considerably modifies light propagation and light-matter interaction. Nanophotonics has become a quickly evolving field, crossing the frontiers of fundamental research to reach real-life applications. Indeed, high-tech nanoscale fabrication processes for dielectric and metal structures, as well as for semi-conductors and composite materials, have paved the way to tailored nano-devices. Potential applications now include a variety of possibilities, such as light-guiding [2], tunable metasurfaces [3], single molecule sensing [4], or cancer treatment processes [5].

As a matter of fact, the physics of light-metal interaction drastically changes at the nanoscale level in comparison with the well known microwave regime. In nanophotonics, the main difference resides in the enormous difference of scale existing between the device and the free space wavelength of the incident field, usually leading to a regime where the scatterer size is comparable to the skin depth. In most cases, a deeply penetrating electromagnetic field will strongly interact with the free electrons of the metal and excite so called plasmonic waves, or plasmons [6], well known from plasma physics [7]. In other words, in these regimes, metals cannot be 
considered to be perfectly conducting, and the electron gas nature of the valence electrons in the metal has to be taken into account, leading to space-time dispersive material laws.

In 1900, Drude proposed a fairly simple yet efficient oscillator model for free electrons in metals [8], becoming a solid reference for theory [6] and experiments [9]. However, this model fails to account for some electronic behaviors, such as transitions between the valence and the conduction band in noble metals, for example. Hence, in order to properly fit experimental permittivity measurements, more sophisticated descriptions emerged, among which the DrudeLorentz model [10], the infinite-oscillator models [11], or general Padé expansions [12].

Although they perform well in most cases, latter refinements remain local models. When the size of a nanostructure decrease beyond approximately $10 \mathrm{~nm}$, the effect of spatial dispersion increases, and must be taken into account within the dispersion model. In such a case, the complexity of the permittivity function significantly increases, leading to a partial differential equation (PDE) in space and time, compared to a system of ordinary differential equations (ODE) for local models. Such descriptions include mutual electron repulsion, hence making the electron behavior dependent not only on the local field value, but also on the neighboring field distribution. For this reason, this model is said to be nonlocal.

Since the impact of nonlocality scales with the effective wave vector [13, 14], gap structures are of particular interest, since they are highly sensitive to spatial dispersion. It should be noted that, when the gap size gets smaller than $1 \mathrm{~nm}$, electron spill-out may occur, and the model considered in this work would fail. Nevertheless, it has been shown that for gap sizes ranging from about $1 \mathrm{~nm}$ to $5 \mathrm{~nm}$, the electronic regime is sensitive to nonlocality while it does not require a spill-out description [15].

\subsection{Numerical challenges}

The simulation of nanophotonic configurations often reveals to be multi-scale problems, because of the strongly different wavelengths occurring due to the plasmonic nature of the metals. As a result, the initial Maxwell problem governing the light propagation, usually in a wavelength regime of several hundreds of nano meters, now has to resolve geometrical details down to $1 \mathrm{~nm}$ leading to an extreme sub-wavelength problem. Additionally, a proper resolution of the geometry is of a crucial importance, thus requiring well adapted mesh sizes in the vicinity of geometric details. Finally, most studies require a wide bandwidth as well as an accurate frequency sampling, in order to detect sharp resonances. In summary, a reliable numerical method for nanophotonics should be geometry conforming, provide accurate results on large bandwidths, and properly discretize small geometric details within large computational domains.

Considering these demands, we believe that a Finite Element Method (FEM) approach is well appropriate due to its flexible mesh capability and high order nature [16]. Broad-band simulations, however, would need a fairly high number of frequency-domain solver calls, guaranteeing a sufficiently fine frequency sampling. For this reason, we have chosen to work in time-domain where only one simulation run is required for an almost arbitrary frequency sampling. Working in time-domain also naturally paves the way to nonlinear dispersion models, which is more difficult to achieve with frequency-domain approaches.

FEM for Maxwell's equations in time-domain requires the inversion of the mass matrix when a time stepping scheme is applied. If for example Nédélec elements are used [17], the matrix structure will be sparse, but not block-diagonal, thus increasing the computational cost of a single time step. Changing the ansatz space to local basis functions leads to a block-diagonal mass matrix, which is efficiently invertible. Local basis functions relax the regularity of the numerical 
solution and leads to discontinuous fields at the cell interfaces. The discontinuity introduced in the approximation induces an increasing amount of discrete unknowns, which is an obvious drawback of the method. It can be overcomed with a parallel implementation strategy exploiting the element-wise locality of the approach. This method is known as the Discontinuous Galerkin Time-Domain (DGTD) method [18]. Busch et al. have intensively pushed the development of DGTD methods purposely tailored for nanophotonics [19], and have also demonstrated the advantages of DGTD in comparison to Finite Difference Time-Domain (FDTD) methods [20].

\subsection{Context and outline of this work}

The present work is a sequel of [21], where we studied the purely nonlocal dispersion model for $2 \mathrm{D}$ configurations. Here, we extend the dispersion model by taking into account different contributions for bound and free electrons: a generalized Padé expansion model is used for the bound electrons, while we use a linearized hydrodynamic model for the description of the nonlocal response of the free electrons (see section 2). Section 3 is concerned with the DGTD formulation, which we extended to upwind fluxes for both Maxwell's equations and the hydrodynamic fluid model. In this context, we provide a semi-discrete stability analysis in section 3.4. Finally, we demonstrate the advantage of using curvilinear elements in non-local computations by means of numerical experiments and computational performance assessments.

\section{Notations and problem statement}

\subsection{Definitions and notations}

For a given open bounded domain $\Omega \subset \mathbb{R}^{3}$, we use standard notations:

- $L^{2}(\Omega)$, the space of square integrable real-valued scalar functions with its canonical scalar product $\langle\cdot, \cdot\rangle_{\Omega}$ and the associated norm $\|\cdot\|_{\Omega} . \mathbf{L}^{2}(\Omega)$ will be its vectorial analogue equipped with the canonical vectorial scalar product (resp. norm) that will be still denoted by $\langle\cdot, \cdot\rangle_{\Omega}$ $\|\cdot\|_{\Omega}\left(\operatorname{resp}\|\cdot\|_{\Omega}\right)$

- $H^{1}(\Omega)$ the space of square integrable functions with square integrable gradient, with $\mathbf{H}^{1}(\Omega)$ its vectorial analogue;

- $H_{0}^{1}(\Omega)$ the space of square integrable functions with square integrable gradient and vanishing trace on the boundary, with $\mathbf{H}_{0}^{1}(\Omega)$ its vectorial analogue;

- $H(\operatorname{curl}, \Omega)$ the space of square integrable functions with square integrable curl;

- $H_{0}(\operatorname{curl}, \Omega)$ the space of square integrable functions with square integrable curl and vanishing tangential trace on the boundary;

- $L^{2}(\partial \Omega)$ with its canonical scalar product $\langle\cdot, \cdot\rangle_{\partial \Omega}$ and the associated norm $\|\cdot\|_{\partial \Omega}$.

Classically, for a given vector field $\mathbf{A}$ of $\mathbb{R}^{3}, \nabla \times \mathbf{A}$, and $\nabla \cdot \mathbf{A}$ respectively denote the curl and the div operator applied to $\mathbf{A}$. 


\subsection{Maxwell's Equations and constitutive laws}

The complete set of macroscopic Maxwell's equations that describe the spatio-temporal evolution of electromagnetic waves on a domain $\Omega \subset \mathbb{R}^{3}$ over a given time interval $[0, T](T>0)$ given are given on $\Omega \times[0, T]$ by (see for example [22])

$$
\begin{array}{ll}
\nabla \times \mathbf{E}=-\partial_{t} \mathbf{B}, & \nabla \times \mathbf{H}=\partial_{t} \mathbf{D}+\mathbf{J}, \\
\nabla \cdot \mathbf{D}=\rho, & \nabla \cdot \mathbf{B}=0,
\end{array}
$$

with $\mathbf{E}, \mathbf{D}, \mathbf{H}, \mathbf{B}, \mathbf{J}: \Omega \times[0, T] \rightarrow \mathbb{R}^{3}$ and $\rho: \Omega \times[0, T] \rightarrow \mathbb{R}$. Here, $\mathbf{E}$ and $\mathbf{H}$ represent the electric and magnetic field, respectively. The magnetic flux density is denoted by $\mathbf{B}$ and the electric displacement and current density respectively by $\mathbf{D}$ and $\mathbf{J}$, and the charge density by $\rho$. These equations are supplemented by constitutive laws linking $\mathbf{D}$ to $\mathbf{E}$ and $\mathbf{B}$ to $\mathbf{H}$ through the introduction of

$$
\mathbf{D}=\varepsilon_{0} \mathbf{E}+\mathbf{P}, \quad \mathbf{B}=\mu_{0} \mathbf{H}+\mathbf{M} .
$$

Here, $\varepsilon_{0}$ and $\mu_{0}$ are the vacuum permittivity and permeability, $\mathbf{P}: \Omega \times[0, T] \rightarrow \mathbb{R}^{3}$ the polarization and $\mathbf{M}: \Omega \times[0, T] \rightarrow \mathbb{R}^{3}$ the magnetization. Throughout the following derivations, non-magnetic materials will be assumed and $\mathbf{M}$ will thus be considered to be zero. Metals are usually seen as a rigid, positive ion cores grid hosting both bound electrons (d-band) and valence electrons (s-band). Hence, the electric polarization $\mathbf{P}$ can be split in two parts: the background polarization of the bound electrons $\mathbf{P}_{\mathrm{b}}$ [23, 13], governing the influence of the background electrons, and $\mathbf{P}_{\mathrm{f}}$, which models the currents in the free electron gas. Exploiting this split, the complete polarization can be written as

$$
\mathbf{P}=\mathbf{P}_{\mathrm{b}}+\mathbf{P}_{\mathrm{f}} .
$$

Multiple models for the modeling of the bound electrons $\mathbf{P}_{\mathrm{b}}$ and free electrons $\mathbf{P}_{\mathrm{f}}$ can be found in literature [11]. In a linear medium, the polarization of the bounded electrons takes into account for the history of the electric field, namely

$$
\mathbf{P}_{b}=\varepsilon_{b} \star_{t} \mathbf{E},
$$

where $\star_{t}$ denotes the convolution in time. Regarding the polarization of the free electrons, one has to take into account for both the history of the field and its variation over space via the relation

$$
\mathbf{P}_{f}=\varepsilon_{f} \star_{(x, t)} \mathbf{E},
$$

where $\star_{(x, t)}$ denotes the convolution in both space and time. We use a generalized dispersion model in order to fit arbitrary bound electron contributions and a quantum hydrodynamic electron response for the free electrons.

\subsection{Generalized dispersion model for bound electrons}

In the frequency-domain (for a given frequency $\omega$ ), equation (2.4) reduces to a multiplication of the frequency dependent quantities associated to $\varepsilon_{b}$ and $\mathbf{E}$. Thus prescribing the expression of a frequency dependent permittivity will determine the model equations (performing an inverse Fourier transform to obtain the time-dependent equations).

In the case of bound electrons, interband transitions can be modeled by Lorentz oscillators motivated by a simple semiquantum model of the form

$$
\varepsilon_{\mathrm{b}}(\omega)=\sum_{i=1}^{k} \frac{f_{i} \omega_{\mathrm{P}}^{2}}{\left(\omega_{i}^{2}-\omega^{2}\right)+i \omega \Gamma_{i}} .
$$




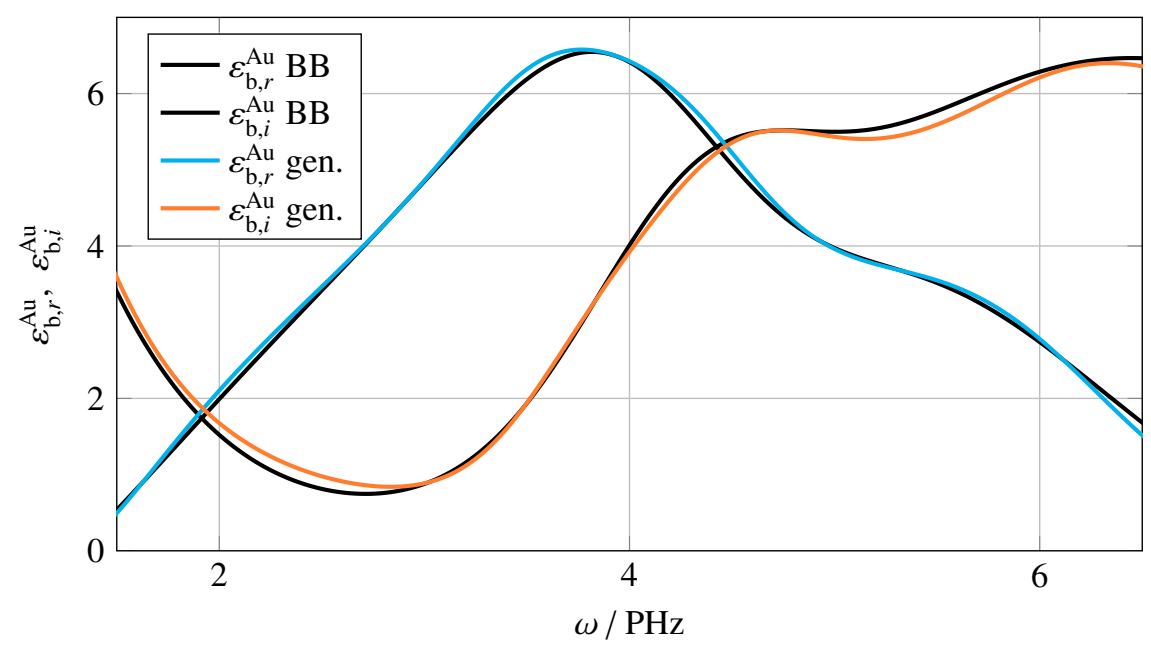

Figure 1: Real and imaginary part of Au's bound permittivity. Black: $\varepsilon_{\mathrm{r}}$ and $\varepsilon_{\mathrm{i}}$ respectively are the real and imaginary part of the experimentally motivated BB model [11]. Colored: the fitted permittivity with our generalized dispersion model [12]. The corresponding fitting coefficients can be found in Table 1.

Here, $\omega_{\mathrm{P}}$ is a physical parameter called the plasma frequency. The other parameters $k$ (the number of poles), $f_{i}, \omega_{i}$ and $\Gamma_{i}$ are degrees of freedom in the model. These will be fixed in order ti fit experimental data of permittivity values. However, this model is limited in precision. As an alternative model, we propose to use the Brendel-Bormann (BB) model applied to a wide range optical frequency range as developed by Rakic et al. [11]. The advantage of this model is that it is able to distinguish between free and bounded electron contributions in the permittivity and thus allows for an accurate description of the latter ones. However, this model demands the evaluation of relatively complex functions, namely the Kummer functions of the second kind (we refer to [11] for details) and is hence complicated to be formulated in the time domain. It turns out that the BB model's permittivities can be nicely fitted by a generalized dispersion model which consists of a Padé series of zero, first and second order poles (see [12])

$$
\varepsilon_{\mathrm{b}}(\omega)=\varepsilon_{\infty}-\frac{\sigma}{i \omega}-\sum_{i \in L_{1}} \frac{a_{i}}{i \omega-b_{i}}-\sum_{i \in L_{2}} \frac{c_{i}-i \omega d_{i}}{\omega^{2}-e_{i}+i \omega f_{i}},
$$

with $\left\{\varepsilon_{\infty}, \sigma, a_{i}, b_{i}, c_{i}, d_{i}, e_{i}, f_{i}\right\} \in \mathbb{R}$ and $L_{1,2}$ being the number of first and second order poles, respectively. These constants are thus fixed in order to provide a good fit of the values given by the BB model. Figure 1 shows an example fit of the bound electron permittivity $\varepsilon_{\mathrm{b}}^{\mathrm{Au}}$ of gold. This fit consists of six second order poles and a constant value $\varepsilon_{\infty}$. Table 1 provides the corresponding parameters. Applying an inverse Fourier transform with the corresponding expression (2.7) yields a system of time domain Ordinary Differential Equations (ODE) driving the evolution of the polarization and polarization current which is then coupled to Maxwell's equations via (2.3). We refer to [24] for more details on this model and to section 2.5 for the global set of equations.

\subsection{Quantum hydrodynamic electron response}

The free electrons of a metal leading to the polarization $\mathbf{P}_{\mathrm{f}}$ can be considered as a free electron gas that is modeled by a nonlinear fluid equation. Given the electron charge $q_{\mathrm{e}}$, its mass $m_{\mathrm{e}}$ and 
Table 1: Fit parameters of Au. Coefficients of the generalized dispersion model (2.7) with $\varepsilon_{\infty}=1.0$ fitted to the BB permittivity of Au. Figure 1 depicts the original BB model and the fitted result.

\begin{tabular}{rrrrr}
\hline $\mathrm{i}$ & $c_{i}\left[\mathrm{~Hz}^{2}\right]$ & $d_{i}[\mathrm{~Hz}]$ & $e_{i}\left[\mathrm{~Hz}^{2}\right]$ & $f_{i}[\mathrm{~Hz}]$ \\
\hline 1 & $0.1244266 \mathrm{E}+32$ & $0.5443769 \mathrm{E}+11$ & $0.1710211 \mathrm{E}+24$ & $0.9838512 \mathrm{E}+15$ \\
2 & $0.1856766 \mathrm{E}+32$ & $0.2936716 \mathrm{E}+12$ & $0.2125471 \mathrm{E}+32$ & $0.1587780 \mathrm{E}+16$ \\
3 & $0.1668009 \mathrm{E}+31$ & $0.3440195 \mathrm{E}+10$ & $0.1643161 \mathrm{E}+31$ & $0.9578440 \mathrm{E}+15$ \\
4 & $0.7350947 \mathrm{E}+24$ & $0.2222866 \mathrm{E}+16$ & $0.1296565 \mathrm{E}+32$ & $0.1400973 \mathrm{E}+16$ \\
5 & $0.3468661 \mathrm{E}+32$ & $0.4572497 \mathrm{E}+11$ & $0.6008717 \mathrm{E}+32$ & $0.1274194 \mathrm{E}+16$ \\
6 & $0.8574585 \mathrm{E}+32$ & $0.8687290 \mathrm{E}+10$ & $0.4155750 \mathrm{E}+32$ & $0.2764158 \mathrm{E}+16$ \\
\hline
\end{tabular}

a damping constant $\gamma$, the nonlinear hydrodynamic electron model reads [25]

$$
m_{\mathrm{e}}\left(\partial_{t}+\mathbf{v} \cdot \nabla\right) \mathbf{v}=-q_{\mathrm{e}}(\mathbf{E}+\mathbf{v} \times \mathbf{B})-m_{\mathrm{e}} \gamma \mathbf{v}-\nabla\left(\frac{\delta G[n]}{\delta n}\right),
$$

together with the continuity equation

$$
\partial_{t} n+\nabla \cdot(n \mathbf{v})=0
$$

where $\mathbf{v}$ represents the fluid velocity, $n$ its density and $q_{\mathrm{e}}(\mathbf{E}+\mathbf{v} \times \mathbf{B})$ is the Lorentz force. The last term of (2.8), containing the quantum pressure $\frac{\delta G[n]}{\delta n}$ eventually determines which quantum mechanical effects of the electron gas is taken into account or left out by an explicit form for the energy functional $G[n]$. Following the discussion by Ciraci [26], this term splits up into a sum of kinetic $(\mathrm{T})$, exchange correlation and potential energy (XC). An approximated functional can be formulated as

$$
G[n] \approx G_{\eta}[n]=T^{\mathrm{TF}}[n]+\frac{1}{\eta} T^{\mathrm{W}}[n]+\mathcal{E}_{\mathrm{XC}},
$$

where the kinetic contribution consists of the Thomas-Fermi (TF) and the von Weizsäcker (W) part.

A fully nonlinear problem as stated in (2.8) is a serious challenge and a reasonably vast range of problems can already be properly modeled by a linearized version of (2.8) [27]. Of course, such a model does not account for nonlinear effects like Second Harmonic Generation (SHG) [28], but already includes spatial dispersion [25, 13, 29, 30]. As a first attempt to study such models, we assume a pure Thomas-Fermi theory and drop the von Weizsäcker contribution as well as the exchange correlation in (2.10) in this work. Further we assume an equilibrium state with zero background velocity, no static electric and magnetic field, and a constant electron density denoted by $n_{0}$. We consider the same expression for the quantum pressure as in [31], which leads to a linear Thomas-Fermi theory $[6,30,13]$. The first order linearized term of the quantum pressure thus expresses as

$$
\beta^{2} \frac{1}{n_{0}} \nabla n
$$

The quantum related parameter $\beta$ has to be chosen according to the physics of the problem. Its choice is a crucial point in this model and it depends on the Fermi velocity $v_{\mathrm{F}}$ and the spatial dimensionality of the problem. Boardman [25] comprehensively discusses the choice of this 
parameter and proposes

$$
\beta=\sqrt{\frac{3 D}{D(D+2)} v_{\mathrm{F}}}
$$

for high frequencies where surface plasmons can be excited [25]. In (2.12), D $=\{1,2,3\}$ is the dimensionality of the Fermi gas. A three dimensional Thomas Fermi gas, as we consider it throughout this work, yields $\beta^{2}=\frac{3}{5} v_{\mathrm{F}}$. Expanding all the fields around an equilibrium state, i.e. $u(\mathbf{r}, t) \approx u_{0}+u_{1}(\mathbf{r}, t), u \in\{n, \mathbf{v}, \mathbf{E}, \mathbf{B}\}$, keeping the linear terms only, and omitting the index $(\cdot)_{1}$ leads to a first order system

$$
\begin{aligned}
m_{\mathrm{e}} \partial_{t} \mathbf{v} & =-q_{\mathrm{e}} \mathbf{E}-m_{\mathrm{e}} \gamma \beta^{2} \frac{1}{n_{0}} \nabla n, \\
\partial_{t} n & =-n_{0} \nabla \cdot \mathbf{v} .
\end{aligned}
$$

Here, we assumed $\partial_{t} n_{0}=\mathbf{v}_{0}=\mathbf{E}_{0}=\mathbf{B}_{0}=0$ (non-moving fluid, absent static electric or magnetic fields, and a constant electron density) and a constant (in both space and time) background electron density $n_{0}$. Differentiating the first equation in (2.13) with respect to the time $t$, exploiting the second equation in (2.13), and using $\mathbf{J}_{\mathrm{f}}=n_{0} q_{\mathrm{e}} \mathbf{v}$, the current density of the unbound electrons in the fluid formally yields

$$
\partial_{t t} \mathbf{J}_{\mathrm{f}}+\gamma \partial_{t} \mathbf{J}_{\mathrm{f}}-\beta^{2} \nabla\left(\nabla \cdot \mathbf{J}_{\mathrm{f}}\right)-\omega_{\mathrm{P}}^{2} \varepsilon_{0} \partial_{t} \mathbf{E}=0,
$$

with $\omega_{\mathrm{P}}=\sqrt{\frac{n_{0} q_{\mathrm{e}}^{2}}{\varepsilon_{0} m_{\mathrm{e}}}}$ being the plasma frequency. Equation (2.14) is a second order PDE in space and time. A direct discretization of (2.14) as is would lead to a so-called variational crime in the DG framework [32], leading us to recast it under a first order system, as follows

$$
\begin{aligned}
\partial_{t} \mathbf{J}_{\mathrm{f}}+\gamma \mathbf{J}_{\mathrm{f}}-\beta^{2} \nabla Q-\omega_{\mathrm{P}}^{2} \varepsilon_{0} \mathbf{E} & =0, \\
\partial_{t} Q-\nabla \cdot \mathbf{J}_{\mathrm{f}} & =0 .
\end{aligned}
$$

System (2.15) can be written as a first order system of hyperbolic PDEs (see next section).

\subsection{Full dispersive Maxwell system}

The split of the bound and free polarization in (2.3) was necessary in order to apply the quantum hydrodynamic electron response model to the free electrons and to allow a flexible model for the bound electrons at the same time. Coupling both to Maxwell's equations at the 
same time leads to the complete system

$$
\begin{aligned}
\nabla \times \mathbf{E}+\mu_{0} \partial_{t} \mathbf{H} & =0, \\
\nabla \times \mathbf{H}-\varepsilon_{0} \varepsilon_{\infty} \partial_{t} \mathbf{E}-\mathbf{J}_{\mathrm{f}}-\mathbf{J}_{\mathrm{b}} & =0, \\
\partial_{t} \mathbf{J}_{\mathrm{f}}+\gamma \mathbf{J}_{\mathrm{f}}-\beta^{2} \nabla Q-\omega_{\mathrm{P}}^{2} \varepsilon_{0} \mathbf{E} & =0, \\
\partial_{t} Q-\nabla \cdot \mathbf{J}_{\mathrm{f}} & =0, \\
-\mathbf{J}_{\mathrm{b}}+\mathcal{J}_{0}+\sum_{i \in L_{1}} \mathcal{J}_{i}+\sum_{i \in L_{2}} \mathcal{J}_{i} & =0, \\
-\mathcal{J}_{0}+\left(\sigma+\sum_{i \in L_{2}} \mathcal{J}_{i}\right) \mathbf{E} & =0, \\
-\mathcal{J}_{i}+a_{i} \mathbf{E}-b_{i} \mathcal{P}_{i} & =0, \quad \forall i \in L_{1}, \\
-\partial_{t} \mathcal{P}_{i}+\mathcal{J}_{i} & =0, \quad \forall i \in L_{1}, \\
-\partial_{t} \mathcal{J}_{i}+\left(c_{i}-d_{i} f_{i}\right) \mathbf{E}-f_{i} \mathcal{J}_{i}-e_{i} \mathcal{P}_{i} & =0, \quad \forall i \in L_{2}, \\
-\partial_{t} \mathcal{P}_{i}+d_{i} \mathbf{E}+\mathcal{J}_{i} & =0, \quad \forall i \in L_{2} .
\end{aligned}
$$

\subsubsection{Boundary conditions}

The set of equations (2.16) is supplemented with initial and boundary conditions. Let us focus on the latter. Regarding the set of pure Maxwell equations, we have to fix boundary conditions for $\mathbf{E}$ and $\mathbf{H}$. We choose not to detail them here since we will use classical boundary conditions that are applied on a perfect electric conducting wall and an absorbing boundary. We do not prescribe any boundary conditions to the set of unknowns issued from the bound electrons, since their evolution is described by a set of ODE's. Let us focus on the set of unknowns issued from the free electrons model, i.e. $\mathbf{J}_{\mathrm{f}}$ and $Q$. We omit the subscript of the free electrons in the following and presume $\mathbf{J}:=\mathbf{J}_{\mathrm{f}}$. System (2.15) can be written as a first order hyperbolic equation as expressed in the third and fourth equation of (2.16). We refer to [21] for the comprehensive discussion and derivation of this system of hyperbolic PDEs; we simply recall the main results for (2.15), in the following. Considering the propagation problem (2.15) on a domain $\Omega$ we claim continuity for the normal component of the polarization current at any sub domain interface between two disjoint domains $\Omega_{1}$ and $\Omega_{2}, \Omega_{1} \cap \Omega_{2}=\partial \Omega_{1,2}$ and $\Omega_{1,2} \subset \Omega$, i.e.

$$
\left.\mathbf{n} \cdot\left(\mathbf{J}_{2}-\mathbf{J}_{1}\right)\right|_{\partial \Omega_{1,2}}=0 .
$$

In the case of $\beta \neq 0$ on $\Omega_{1}$ and $\beta \equiv 0$ on $\Omega_{2}, \partial \Omega_{1,2}$ becomes a boundary of the nonlocal domain. Supposing a vanishing polarization current outside the nonlocal domain $\Omega_{1}$, the resulting boundary condition reads

$$
\left.\mathbf{n} \cdot \mathbf{J}_{1}\right|_{\partial \Omega_{1,2}}=0 .
$$

In the special case of $\Omega_{1}=\Omega$, (2.18) formulates the boundary condition for the entire boundary $\partial \Omega$ of the nonlocal domain $\Omega$.

\subsubsection{Study of the hyperbolicity}

If we focus on the Maxwell-hydrodynamical part of (2.16) (i.e. the first four equations, neglecting the ODE's contributions), one can study its hyperbolicity. We here recall the associated 
eigenvalues and again refer to [21] for details. For $\xi=\left\{\xi_{1}, \xi_{2}, \xi_{3}\right\}^{T} \in \mathbb{R}^{3}$, the eigenvalues of the Maxwell-hydrodynamic system are given by

$$
\lambda(\|\xi\|)=\left\{\begin{array}{llllllllll}
0 & 0 & 0 & 0 & -c_{r}\|\xi\| & -c_{r}\|\xi\| & c_{r}\|\xi\| & c_{r}\|\xi\| & -\beta\|\xi\| & \beta\|\xi\|
\end{array}\right\} .
$$

These will be of importance when discussing the numerical fluxes in the numerical sections that follow.

\subsubsection{Energy}

The considered system (2.16) benefits from an energy principle. Indeed, we can formulate the total energy as a sum of the local and nonlocal contribution as

$$
\mathcal{E}=\mathcal{E}_{\mathrm{gen}}+\mathcal{E}_{\mathrm{NL}},
$$

with

$$
\begin{array}{r}
\mathcal{E}_{\text {gen }}(t)=\frac{1}{2}\left(\mu_{0}\|\mathbf{H}(t)\|_{\Omega}^{2}+\varepsilon_{\infty} \varepsilon_{0}\|\mathbf{E}(t)\|_{\Omega}^{2}+\sum_{l \in L_{1}} \frac{b_{l}}{a_{l}}\left\|\mathcal{J}_{l}(t)\right\|_{\Omega}^{2}+\right. \\
\left.\sum_{l \in L_{2}} \frac{e_{l}}{c_{l}+d_{l} f_{l}}\left\|\boldsymbol{P}_{l}(t)\right\|_{\Omega}^{2}+\sum_{l \in L_{2}} \frac{1}{c_{l}+d_{l} f_{l}}\left\|\mathcal{J}_{l}(t)\right\|_{\Omega}^{2}\right)
\end{array}
$$

and

$$
\mathcal{E}_{\mathrm{NL}}(t):=\frac{1}{2}\left(\mu_{0} \omega_{\mathrm{P}}^{2} \varepsilon_{0}\|\mathbf{H}(t)\|_{\Omega}^{2}+\varepsilon_{\infty} \varepsilon_{0}^{2} \omega_{\mathrm{P}}^{2}\|\mathbf{E}(t)\|_{\Omega}^{2}+\|\mathbf{J}(t)\|_{\Omega}^{2}+\beta^{2}\|Q(t)\|_{\Omega}^{2}\right),
$$

Assuming the boundary condition (2.18) for the hydrodynamic part and perfectly electric conducting (PEC) for Maxwell's equations, leads to a bounded energy which is either strictly preserved or monotonically decreasing if damping is taken into account. Combining arguments from [24] (for the generalized model) and [21] (for the linearized hydrodynamic model), we deduce that

$$
\partial_{t} \mathcal{E}(t)=\partial_{t} \mathcal{E}_{\mathrm{gen}}(t)+\partial_{t} \mathcal{E}_{\mathrm{NL}}(t) \leq \max _{l \in L_{2}} d_{l} \sqrt{\frac{e_{l}}{\varepsilon_{\infty} \varepsilon_{0}\left(c_{l}+d_{l} f_{l}\right)}} \mathcal{E}(t),
$$

that, using a Grönwall type Lemma, implies the boundedness of the energy. Let us point out that if the coefficients $d_{l}$ or $e_{l}$ vanish, then the energy is decreasing.

\section{Numerical scheme}

In the previous section, we set up the physical framework and detailed the considered physical model (2.16). This section describes the spatial discretization of the latter based on a DG formulation. For the sake of simplicity, we focus within this section on the PDE character of system (2.16) avoiding to explicitly write up the generalized dispersion contribution as well. We ask the reader to always keep the missing ODEs from (2.16) in mind.

The DG method is based on a local weak formulation, that will require numerical fluxes in order to treat the arising discontinuities. In this work, we extend our previous work [21] by several means. First, the scheme used extend the possible numerical traces by including upwind fluxes instead of centered fluxes and we demonstrate its semi-discrete stability. Furthermore, the explicit time integration of the obtained semi-discrete scheme is now performed by a Low Storage Runge-Kutta (LSRK) scheme. Finally, we extend the use of curvilinear elements from a previous work [33] in order to guarantee the high order nature of our scheme even if complex geometries are of concern. 


\subsection{The Discontinuous Galerkin method}

We now want to apply the DG method to system (2.15), which is done in the following steps: (i) defining a weak formulation, (ii) choosing an appropriate finite element space for the basis functions, and eventually (iii) evaluating the resulting integrals from the discrete weak Galerkin formalism and semi-discrete scheme. Here, semi-discrete means discrete in space and continuous in time.

\subsection{Weak formulation}

The starting point of the DG formulation is a local weak formulation of system (2.15). Spatial discretization with the DG method requires a special choice of finite element spaces and thus of polynomial basis functions on a given mesh. This choice clearly distinguishes DG methods from continuous FEM. While Nédélec based FEM use basis function from conforming discretization spaces, DG relies on element-wise local basis functions. The use of local basis functions implies that the continuity of the fields is not enforced and discontinuities at the cell interfaces may arise. The treatment of those discontinuities provides an additional design parameter for the final algorithm. As we will see later, the discontinuities at the mesh element interfaces will be handled by a numerical flux. Further, the choice of this flux is not unique and will strongly influence numerical properties such as the numerical dissipation and the existence of spurious solutions [19]. Different polynomial basis expansions are possible, but in this work, we concentrate on nodal Lagrange based basis functions on a tetrahedral mesh. Let us suppose that the computational domain $\Omega$ can be discretized as a conformal, quasi-uniform tetrahedral mesh as $\bar{\Omega}=\bigcup_{\forall i \in \mathcal{N}_{\Omega}} \bar{\Omega}_{i}$, with $\mathcal{N}_{\Omega}$ being the set of indices of mesh elements and $\Omega_{i}$ the tetrahedra with a characteristic size parameter $h>0$. Furthermore, $\forall i \in \mathcal{N}_{\Omega}, \mathcal{N}_{\Omega_{i}}$ denotes the set of indices of the neighboring elements of $\Omega_{i}$ (having a face in common) and $s_{i q}=\bar{\Omega}_{i} \cap \bar{\Omega}_{q}, \forall q \in \mathcal{N}_{\Omega_{i}}$, the set of internal faces. We define the approximation space

$$
\mathcal{V}_{h}^{p}(\Omega):=\left\{v \in \mathbf{L}^{2}(\Omega),\left.v\right|_{\Omega_{i}} \in \mathbf{P}_{p}\left(\Omega_{i}\right), \forall i \in \mathcal{N}_{\Omega}\right\}
$$

where $\mathbf{P}_{p}\left(\Omega_{i}\right)$ is the space of polynomials of maximum degree $p \in \mathbb{N}$ on $\Omega_{i} \cdot \mathcal{V}_{h}^{p}(\Omega)$ is a finite dimensional subspace of $\mathbf{L}^{2}(\Omega)$. We choose a polynomial basis of $\mathcal{V}_{h}^{p}(\Omega)$ formed by polynomial functions $\left(\phi_{i j}\right)_{\mathcal{N}_{\Omega} \times\left[1, P_{i}\right]}$, with $P_{i}$ the number of Degrees of Freedom (DoF) for the i-th element. For any $\mathbf{A} \in\left(\mathcal{V}_{h}^{p}(\Omega)\right)^{3}$, we denote by $\mathbf{A}_{i}$ the restriction of $\mathbf{A}$ to $\Omega_{i}$ (analogous definition holds for a scalar field of $\mathcal{V}_{h}^{p}(\Omega)$ ).

We now turn to the formulation of the DG method. We express the discrete weak formulation locally on each element of the mesh and perform an integration by part and derive a local Galerkin formulation as follows: Find $(\mathbf{E}, \mathbf{H}, Q, \mathbf{J}) \in\left(\mathcal{V}_{h}^{p}(\Omega)\right)^{10}$, such that for all $i \in \mathcal{N}_{\Omega}, k \in \llbracket 0, P_{i} \rrbracket$ :

$$
\begin{aligned}
\mu_{0} \partial_{t}<\mathbf{H}_{i}, \boldsymbol{\phi}_{i k}>_{\Omega_{i}}= & -<\mathbf{E}_{i}, \nabla \times \boldsymbol{\phi}_{i k}>_{\Omega_{i}}-<\mathbf{E}_{i}^{*} \times \boldsymbol{\phi}_{i k}, \mathbf{n}>_{\partial \Omega_{i}}, \\
\varepsilon_{0} \varepsilon_{\infty} \partial_{t}<\mathbf{E}_{i}, \boldsymbol{\phi}_{i k}>_{\Omega_{i}}= & <\mathbf{H}_{i}, \nabla \times \boldsymbol{\phi}_{i k}>_{\Omega_{i}}+<\mathbf{H}_{i}^{*} \times \boldsymbol{\phi}_{i k}, \mathbf{n}>_{\partial \Omega_{i}}-<\mathbf{J}_{i}, \boldsymbol{\phi}_{i k}>_{\Omega_{i}}, \\
\partial_{t}<\mathbf{J}_{i}, \boldsymbol{\phi}_{i k}>_{\Omega_{i}}= & -\beta^{2}<Q_{i}, \nabla \cdot \boldsymbol{\phi}_{i k}>_{\Omega_{i}}+\beta^{2}<Q_{i}^{*} \boldsymbol{\phi}_{i k}, \mathbf{n}>_{\partial \Omega_{i}} \\
& -\gamma<\mathbf{J}_{i}, \boldsymbol{\phi}_{i k}>_{\Omega_{i}}+\omega_{\mathrm{P}}^{2} \varepsilon_{0}<\mathbf{E}_{i}, \boldsymbol{\phi}_{i k}>_{\Omega_{i}}, \\
\partial_{t}<Q_{i}, \phi_{i k}>_{\Omega_{i}}= & -<\mathbf{J}_{i}, \nabla \phi_{i k}>_{\Omega_{i}}+<\mathbf{J}_{i}^{*} \phi_{i k}, \mathbf{n}>_{\partial \Omega_{i}},
\end{aligned}
$$


with $\boldsymbol{\phi}_{i j}^{u}:=\phi_{i j} \mathbf{e}_{u}$, where $u \in\{1,2,3\}$ refers to the spatial variable / coordinate axis. Since the field values at a cell interface are not unequivocal (the unknowns can refer to the value on either side of the interface), we introduce the star notation $(\cdot)^{*}$ that makes reference to the numerical flux on the cell boundary $\partial \Omega_{i}$. This flux is used to recover a proper definition of the surface integrals, and shall be detailed in the next section. Using the decomposition of each field on the basis, one can rewrite (3.2) in a matrix form. We refer to [21] for a detailed derivation of the corresponding mass and flux matrices.

\subsection{Numerical fluxes}

As previously mentioned, each cell has strictly local basis functions, no continuity of the fields is ensured between two neighboring cells, and the definition of the surface integrals present in (3.2) is ambiguous. In order to overcome this dilemma, a numerical flux is used which weakly reinforce the tangential continuity of the solution. Finding the appropriate numerical flux is subject to solving a Riemann problem at each cell interface. Since this is very classical, we skip the details of the derivation of the numerical flux for Maxwell's equations and refer to the literature $[18,19,12]$ for details. For our purposes let us recall the general upwind flux for $\mathbf{E}$ and $\mathbf{H}$ fields

$$
\begin{aligned}
\mathbf{E}_{*}^{-} & =\frac{1}{Y^{-}+Y^{+}}\left(\{Y \mathbf{E}\}_{-+}-\alpha\left(\mathbf{n}_{-+} \times \llbracket \mathbf{H} \rrbracket_{-+}\right)\right), \\
\mathbf{H}_{*}^{-} & =\frac{1}{Z^{-}+Z^{+}}\left(\{Z \mathbf{H}\}_{-+}+\alpha\left(\mathbf{n}_{-+} \times \llbracket \mathbf{E} \rrbracket_{-+}\right)\right),
\end{aligned}
$$

as well as

$$
\left.\mathbf{E}_{*}^{-}\right|_{\partial \Omega_{\mathrm{PEC}}}=0,
$$

for the Perfectly Electrical Conducting (PEC) boundary faces and

$$
\begin{aligned}
\left.\mathbf{E}_{*}^{-}\right|_{\partial \Omega_{\mathrm{ABC}}} & =\frac{1}{2}\left(\mathbf{E}_{-}-Z^{-}\left(\mathbf{n}_{-+} \times \mathbf{H}_{-}\right)\right), \\
\left.\mathbf{H}_{*}^{-}\right|_{\partial \Omega_{\mathrm{ABC}}} & =\frac{1}{2}\left(\mathbf{H}_{-}+Y^{-}\left(\mathbf{n}_{-+} \times \mathbf{E}_{-}\right)\right),
\end{aligned}
$$

for the Absorbing Boundary Condition (ABC). Here, $Z=\sqrt{\mu / \varepsilon}$ and $Y=\sqrt{\varepsilon / \mu}$ respectively are the impedance and admittance of the left or right cell of the interface denoted by ' $-+{ }^{\prime}$ with $\mathbf{n}_{-+}=-\mathbf{n}_{+-}$and $\alpha \in[0,1]$ weights the upwinding. The case $\alpha=0$ is called centered flux and in the case $\alpha=1$ the scheme is fully upwind. Means and jumps at the cell interface are defined by

$$
\{\mathbf{A}\}_{-+}:=\mathbf{A}^{-}+\mathbf{A}^{+}, \llbracket \mathbf{A} \rrbracket_{-+}:=\mathbf{A}^{-}-\mathbf{A}^{+} .
$$

Regarding the hydrodynamic part $\mathbf{J}$ and $Q$, centered fluxes have already been considered in [21], and we now propose to derive the formulation of the upwind fluxes. Let us ignore the coupling to Maxwell's equations, neglect the damping term in (2.15), and focus on the hydrodynamic equation

$$
\begin{aligned}
\frac{1}{\beta^{2}} \partial_{t} \mathbf{J} & =\nabla Q \\
\partial_{t} Q & =\nabla \cdot \mathbf{J} .
\end{aligned}
$$


Proposition 3.1. The upwind flux for (3.7) is given by

$$
\begin{aligned}
Q_{\star}^{-} & =\frac{1}{\beta^{-}+\beta^{+}}\left(\{\beta Q\}_{-+}-\alpha\left(\mathbf{n} \cdot \llbracket \mathbf{J} \rrbracket_{-+}\right)\right), \\
\mathbf{n} \cdot \mathbf{J}_{\star}^{-} & =\frac{\beta^{-} \beta^{+}}{\beta^{-}+\beta^{+}}\left(\mathbf{n} \cdot\left\{\frac{\mathbf{J}}{\beta}\right\}_{-+}-\alpha \llbracket Q \rrbracket_{-+}\right),
\end{aligned}
$$

and on the boundary of the nonlocal dispersion domain

$$
\begin{aligned}
\left.Q_{\star}^{-}\right|_{\partial \Omega_{\mathrm{NL}}} & =Q^{-}, \\
\left.\mathbf{n} \cdot \mathbf{J}_{\star}^{-}\right|_{\partial \Omega_{\mathrm{NL}}} & =0 .
\end{aligned}
$$

Proof. Casting (3.7) into a conservative form leads to

$$
Q \partial_{t} \mathcal{W}+\nabla \cdot \mathcal{F}(\mathcal{W})=0
$$

with

$$
\begin{aligned}
& \mathcal{W}=\left[\begin{array}{l}
\mathbf{J}_{x} \\
\mathbf{J}_{y} \\
\mathbf{J}_{z} \\
Q
\end{array}\right], \quad Q=\operatorname{diag}\left\{\beta^{-2}, \beta^{-2}, \beta^{-2}, 1\right\}, \\
& \mathcal{F}_{x}=\left[\begin{array}{c}
-Q \\
0 \\
0 \\
-\mathbf{J}_{x}
\end{array}\right], \quad \mathcal{F}_{y}=\left[\begin{array}{c}
0 \\
-Q \\
0 \\
-\mathbf{J}_{y}
\end{array}\right], \quad \mathcal{F}_{z}=\left[\begin{array}{c}
0 \\
0 \\
-Q \\
-\mathbf{J}_{z}
\end{array}\right] .
\end{aligned}
$$

Following the notations used in [12], the Rankine-Hugoniot jump conditions read

$$
\begin{aligned}
\beta^{-} Q^{-}\left(\mathcal{W}_{\star}^{-}-\mathcal{W}^{-}\right)+\mathbf{n}_{-+} \cdot\left(\mathcal{F}_{\star}^{-}-\mathcal{F}^{-}\right) & =0, \\
\mathbf{n}_{-+} \cdot\left(\mathcal{F}_{\star}^{-}-\mathcal{F}_{\star}^{+}\right) & =0, \\
-\beta^{+} Q^{+}\left(\mathcal{W}_{\star}^{+}-\mathcal{W}^{+}\right)+\mathbf{n}_{-+} \cdot\left(\mathcal{F}_{\star}^{+}-\mathcal{F}^{+}\right) & =0 .
\end{aligned}
$$

Here, we have used the eigenvalues that correspond to the hydrodynamic part in (2.15) as detailed in [21]. For the sake of simplicity, we omit the direction of $\mathbf{n}_{-+}$and fix the normal vector to point from "-" to "+", i.e. $\mathbf{n}:=\mathbf{n}_{-+}$. Summing up the second and third equation of (3.12) gives

$$
\begin{aligned}
\beta^{-} Q^{-}\left(\mathcal{W}_{\star}^{-}-\mathcal{W}^{-}\right)+\mathbf{n} \cdot\left(\mathcal{F}_{\star}^{-}-\mathcal{F}^{-}\right) & =0, \\
-\beta^{+} Q^{+}\left(\mathcal{W}_{\star}^{+}-\mathcal{W}^{+}\right)+\mathbf{n} \cdot\left(\mathcal{F}_{\star}^{-}-\mathcal{F}^{+}\right) & =0 .
\end{aligned}
$$

Then, respectively multiplying with $\beta^{+} Q^{+}$and $\beta^{-} Q^{-}$(that are commuting), and summing yields

$$
\begin{aligned}
& \beta^{-} \beta^{+} Q^{-} Q^{+}\left(\mathcal{W}_{\star}^{-}-\mathcal{W}^{-}-\mathcal{W}_{\star}^{+}+\mathcal{W}^{+}\right) \\
&+\left(\beta^{-} Q^{-}+\beta^{+} Q^{+}\right) \mathbf{n} \cdot \mathcal{F}_{\star}^{-} \\
&-\beta^{-} Q^{-} \mathbf{n} \cdot \mathcal{F}^{+}-\beta^{+} Q^{+} \mathbf{n} \cdot \mathcal{F}^{-}=0 . \\
& 12
\end{aligned}
$$


The normal flux is given by

$$
\mathbf{n} \cdot \mathcal{F}=\left[\begin{array}{c}
-\mathbf{n} Q \\
-\mathbf{n} \cdot \mathbf{J}
\end{array}\right] .
$$

Inserting this flux in (3.14) gives for the $\mathbf{J}$ part

$$
\begin{gathered}
\beta^{-} \beta^{+} \frac{1}{\left(\beta^{+}\right)^{2}} \frac{1}{\left(\beta^{-}\right)^{2}}\left(\mathbf{J}_{\star}^{-}-\mathbf{J}^{-}-\mathbf{J}_{\star}^{+}+\mathbf{J}^{+}\right) \\
+\left(\frac{1}{\beta^{+}}+\frac{1}{\beta^{-}}\right) \mathbf{n}\left(-Q_{\star}^{-}\right) \\
+\frac{1}{\beta^{+}} \mathbf{n}\left(Q^{-}\right)+\frac{1}{\beta^{-}} \mathbf{n}\left(Q^{+}\right)=0 .
\end{gathered}
$$

Performing the cross product with $\mathbf{n}$ and together with (3.12) yields

$$
\begin{aligned}
\mathbf{n} \times\left(\mathbf{J}_{\star}^{-}-\mathbf{J}_{\star}^{+}\right) & =\mathbf{n} \times\left(\mathbf{J}^{-}-\mathbf{J}^{+}\right), \\
\mathbf{n} \cdot\left(\mathbf{J}_{\star}^{-}-\mathbf{J}_{\star}^{+}\right) & =0 .
\end{aligned}
$$

The vector identity $\mathbf{A}=(\mathbf{n} \cdot \mathbf{A}) \mathbf{n}-\mathbf{n} \times(\mathbf{n} \times \mathbf{A})$ applied to $\mathbf{A}=\mathbf{J}_{\star}^{-}-\mathbf{J}_{\star}^{+}$reads

$$
\begin{aligned}
& \mathbf{J}_{\star}^{-}-\mathbf{J}_{\star}^{+}=\underbrace{\left(\mathbf{n} \cdot\left(\mathbf{J}_{\star}^{-}-\mathbf{J}_{\star}^{+}\right)\right)}_{=0} \mathbf{n}-\mathbf{n} \times\left(\mathbf{n} \times\left(\mathbf{J}_{\star}^{-}-\mathbf{J}_{\star}^{+}\right)\right), \\
& \mathbf{J}_{\star}^{-}-\mathbf{J}_{\star}^{+}=-\mathbf{n} \times\left(\mathbf{n} \times\left(\mathbf{J}_{\star}^{-}-\mathbf{J}_{\star}^{+}\right)\right),
\end{aligned}
$$

and similarly for $\mathbf{A}=\mathbf{J}^{-}-\mathbf{J}^{+}$

$$
\begin{aligned}
& \mathbf{J}^{-}-\mathbf{J}^{+}=\left(\mathbf{n} \cdot\left(\mathbf{J}^{-}-\mathbf{J}^{+}\right)\right) \mathbf{n}-\mathbf{n} \times\left(\mathbf{n} \times\left(\mathbf{J}^{-}-\mathbf{J}^{+}\right)\right), \\
& \mathbf{J}_{\star}^{-}-\mathbf{J}_{\star}^{+}=\mathbf{J}^{-}-\mathbf{J}^{+}-\left(\mathbf{n} \cdot\left(\mathbf{J}^{-}-\mathbf{J}^{+}\right)\right) \mathbf{n} .
\end{aligned}
$$

Plugging (3.19) into (3.16) leads to

$$
\begin{aligned}
& \mathbf{n} Q_{\star}^{-}=\frac{1}{\beta^{-}+\beta^{+}}\left(\mathbf{n}\left(Q^{-} \beta^{-}+Q^{+} \beta^{+}\right)-\mathbf{n}\left(\mathbf{n} \cdot\left(\mathbf{J}^{-}-\mathbf{J}^{+}\right)\right)\right), \\
& \mathbf{n} Q_{\star}^{-}=\frac{1}{\beta^{-}+\beta^{+}}\left(\mathbf{n}\{\beta Q\}_{-+}-\mathbf{n}\left(\mathbf{n} \cdot \llbracket \mathbf{J} \rrbracket_{-+}\right)\right) .
\end{aligned}
$$

Similar steps for the $Q$ part lead to

$$
\begin{aligned}
& \beta^{-} \beta^{+}\left(Q_{\star}^{-}-Q^{-}-Q_{\star}^{+}+Q^{+}\right) \\
&-\left(\beta^{-}+\beta^{+}\right) \mathbf{n} \cdot \mathbf{J}_{\star}^{-} \\
&+\beta^{-} \mathbf{n} \cdot \mathbf{J}^{+}+\beta^{+} \mathbf{n} \cdot \mathbf{J}^{-}=0 .
\end{aligned}
$$

that with (3.12) simplifies to

$$
\begin{aligned}
-\mathbf{n} \cdot \mathbf{J}_{\star}^{+} & =-\frac{1}{\beta^{-}+\beta^{+}}\left(\mathbf{n} \cdot\left(\beta^{+} \mathbf{J}^{-}+\beta^{-} \mathbf{J}^{+}\right)-\beta^{-} \beta^{+}\left(Q^{-}-Q^{+}\right)\right), \\
\mathbf{n} \cdot \mathbf{J}_{\star}^{+} & =\frac{\beta^{-} \beta^{+}}{\beta^{-}+\beta^{+}}\left(\mathbf{n} \cdot\left\{\frac{\mathbf{J}}{\beta}\right\}_{13}-\llbracket Q \rrbracket_{-+}\right) .
\end{aligned}
$$


If we now allow the contribution of the jump value $\llbracket \cdot \rrbracket$ to be tunable by a real tuning parameter $\alpha \in[0,1]$ we obtain (3.8).

For $\alpha=0$ we retain the centered flux as it has been used in [21] and $\alpha=1$ yields a fully upwind scheme. The eventual choice of $\alpha$ heavily influences the numerical properties of the final discretized version of (2.15). Properties like numerical dissipation and the occurrence of spurious solutions are particularly sensitive to the fluxes as we will see later in section 4 on Mie scattering and the spherical dimer system.

\subsection{Semi-discrete stability}

We define the semi-discrete energy through element-wise semi-discrete energies that mimic (2.20) a replacement of the continuous fields by their semi-discrete ones

$$
\mathcal{E}_{h}(t)=\mathcal{E}_{h, N L}(t)+\mathcal{E}_{h, g e n}(t)
$$

where $\mathcal{E}_{h, N L}(t)=\sum_{i \in N_{\Omega}}\left(\mathcal{E}_{h, N L}\right)_{i}(t)$ and $\mathcal{E}_{h, g e n}(t)=\sum_{i \in N_{\Omega}}\left(\mathcal{E}_{h, g e n}\right)_{i}(t)$. The element-wise $\left(\mathcal{E}_{h, N L}\right)_{i}$ and $\left(\mathcal{E}_{h, g e n}\right)_{i}$ are obtained by replacing $\Omega$ with $\Omega_{i}$ and every continuous field with its semi-discrete counterpart in the continuous expressions defined previously. We here extend the stability results to more general boundary conditions and fluxes in comparison to both [24] and [21].

Proposition 3.2. Let us suppose that $\beta$ is constant on $\Omega$ and that the semi-discrete fields are $C^{1}$ in time. The semi-discrete energy is bounded on $[0, T]$. The semi-discrete scheme is thus stable.

Proof. We first focus on the nonlocal model's energy contribution. In order to keep the question of boundary conditions as general as possible, we will consider that the faces of the mesh that intersect the boundary of the domain could be either subject to PEC boundary conditions or ABC (via a Silver Müller approximation). Performing the time derivative and using the semi-discrete equations lead to the element-wise change of the semi-discrete energy

$$
\begin{aligned}
\partial_{t}\left(\mathcal{E}_{h, \mathrm{NL}}\right)_{i}(t)= & \frac{1}{2}\left(\varepsilon_{0} \omega_{\mathrm{P}}^{2} A_{i}+\beta^{2} B_{i}-\gamma\left\|\mathbf{J}_{i}\right\|_{\Omega_{i}}^{2}\right), \\
A_{i}:= & -<\mathbf{E}_{i}, \nabla \times \mathbf{H}_{i}>_{\Omega_{i}}-<\mathbf{E}^{*} \times \mathbf{H}_{i}, \mathbf{n}>_{\partial \Omega_{i}} \\
& +<\mathbf{H}_{i}, \nabla \times \mathbf{E}_{i}>_{\Omega_{i}}+<\mathbf{H}^{*} \times \mathbf{E}_{i}, \mathbf{n}>_{\partial \Omega_{i}}, \\
B_{i}:= & -<Q_{i}, \nabla \cdot \mathbf{J}_{i}>_{\Omega_{i}}+<Q^{*} \mathbf{J}_{i}, \mathbf{n}>_{\partial \Omega_{i}} \\
& -<\mathbf{J}_{i}, \nabla Q_{i}>_{\Omega_{i}}+<\mathbf{J}^{*} Q_{i}, \mathbf{n}>_{\partial \Omega_{i}} .
\end{aligned}
$$

For the sake of easier readability we will drop the subscript ()$_{h}$ for semi-discrete quantities and we assume $Z=Y=\beta=1$ without loss of generality in the scope of this proof. Integration by parts leads to a purely surfacic expression for $A_{i}$

$$
\begin{aligned}
A_{i}= & \frac{1}{2} \sum_{q} \int_{s_{i q}}\left(\mathbf{H}_{q} \times \mathbf{E}_{i}\right) \cdot \mathbf{n}_{i q}-\left(\mathbf{E}_{q} \times \mathbf{H}_{i}\right) \cdot \mathbf{n}_{i q}- \\
& \frac{\alpha}{2} \sum_{q} \int_{s_{i q}}\left(\left(\mathbf{n}_{i q} \times \mathbf{E}_{q}\right) \times \mathbf{E}_{i}\right) \cdot \mathbf{n}_{i q}+\left(\left(\mathbf{n}_{i q} \times \mathbf{H}_{q}\right) \times \mathbf{H}_{i}\right) \cdot \mathbf{n}_{i q}+ \\
& \frac{\alpha}{2} \sum_{q} \int_{s_{i q}}\left(\left(\mathbf{n}_{i q} \times \mathbf{E}_{i}\right) \times \mathbf{E}_{i}\right) \cdot \mathbf{n}_{i q}+\left(\left(\mathbf{n}_{i q} \times \mathbf{H}_{i}\right) \times \mathbf{H}_{i}\right) \cdot \mathbf{n}_{i q}+\mathfrak{a}_{i}^{\text {boundary }} .
\end{aligned}
$$


Here $\mathfrak{a}_{i}^{\text {boundary }}$ contains the contributions of the faces of the mesh elements that belong to the boundary (either PEC or ABC). In the case of the cell $i$ being an inner cell, the contribution of $\mathfrak{a}_{i}^{\text {boundary }}$ is obviously vanishing. We denote by $\mathcal{F}^{\text {int }}$ the set of all internal faces, $\mathcal{F}^{\text {PEC }}$ the set of all PEC faces, $\mathcal{F}^{\mathrm{ABC}}$ the set of all $\mathrm{ABC}$ faces. Performing the sum over all faces yields

$$
\begin{array}{r}
A=\alpha \sum_{s_{i q} \in \mathcal{F}^{\text {int }}} \int_{s_{i q}}-\left(\left(\mathbf{n}_{i q} \times \mathbf{E}_{q}\right) \times \mathbf{E}_{i}\right) \cdot \mathbf{n}_{i q}-\left(\left(\mathbf{n}_{i q} \times \mathbf{H}_{q}\right) \times \mathbf{H}_{i}\right) \cdot \mathbf{n}_{i q}+ \\
\frac{1}{2}\left[\left(\left(\mathbf{n}_{i q} \times \mathbf{E}_{i}\right) \times \mathbf{E}_{i}\right) \cdot \mathbf{n}_{i q}+\left(\left(\mathbf{n}_{i q} \times \mathbf{H}_{i}\right) \times \mathbf{H}_{i}\right) \cdot \mathbf{n}_{i q}+\right. \\
\left.\quad\left(\left(\mathbf{n}_{i q} \times \mathbf{E}_{q}\right) \times \mathbf{E}_{q}\right) \cdot \mathbf{n}_{i q}+\left(\left(\mathbf{n}_{i q} \times \mathbf{H}_{q}\right) \times \mathbf{H}_{q}\right) \cdot \mathbf{n}_{i q}\right] \\
+\sum_{s_{i} \in \mathcal{F}^{\mathrm{PEC}}} \mathfrak{a}_{i}^{\text {boundary }}+\sum_{s_{i} \in \mathcal{F} \mathrm{ABC}} \mathfrak{a}_{i}^{\text {boundary }} .
\end{array}
$$

If we denote $c^{\mathrm{PEC}}:=\sum_{s_{i} \in \mathcal{F}^{\mathrm{PEC}}} \mathfrak{a}_{i}^{\text {boundary }}$ and $c^{\mathrm{ABC}}:=\sum_{s_{i} \in \mathcal{F}^{\mathrm{ABC}}} \mathfrak{a}_{i}^{\text {boundary }}$, equation (3.26) can be cast into a simpler form

$$
A=-\frac{\alpha}{2}\left(\left\|\mathbf{n} \times \llbracket \mathbf{E}_{h} \rrbracket\right\|_{\mathcal{F}^{\text {int }}}^{2}+\left\|\mathbf{n} \times \llbracket \mathbf{H}_{h} \rrbracket\right\|_{\mathcal{F}^{\text {int }}}^{2}\right)+\mathfrak{a}^{\mathrm{PEC}}+\mathfrak{a}^{\mathrm{ABC}} .
$$

Using similar arguments as for the internal faces and using the fluxes on the boundary faces yields

$$
\mathfrak{a}^{\mathrm{PEC}}=-\alpha\left\|\mathbf{n} \times \mathbf{E}_{h}\right\|_{\mathcal{F} \text { ext }}^{2}=0 .
$$

Regarding the first order Silver-Müller absorbing boundary condition, i.e. ABC, which is imposed by a modified numerical flux as mentioned in the section describing fluxes, one obtains

$$
\begin{aligned}
& \mathfrak{a}^{\mathrm{ABC}}= \frac{1}{2} \sum_{s_{i q} \in \mathcal{F}^{A B C}} \int_{s_{i q}}\left(\mathbf{E}_{i} \times \mathbf{H}_{i}\right) \cdot \mathbf{n}_{i q}-\left(\mathbf{H}_{i} \times \mathbf{E}_{i}\right) \cdot \mathbf{n}_{i q}+ \\
& \mathbf{E}_{i} \cdot\left(\mathbf{n}_{i q} \times\left(\mathbf{H}_{i}+\mathbf{n} \times \mathbf{E}_{i}\right)\right)-\mathbf{H}_{i} \cdot\left(\mathbf{n}_{i q} \times\left(\mathbf{E}_{i}-\mathbf{n} \times \mathbf{H}_{i}\right)\right) \\
&=-\frac{1}{2}\left(\left\|\mathbf{n} \times \mathbf{E}_{h}\right\|_{\mathcal{F} \mathrm{ABC}}^{2}+\left\|\mathbf{n} \times \mathbf{H}_{h}\right\|_{\mathcal{F} \mathrm{ABC}}^{2}\right) .
\end{aligned}
$$

Using similar arguments for the hydrodynamic part $B_{i}$ (splitting, summing over faces, denoting 
$\mathrm{b}^{\mathrm{ext}}$ the contribution of all boundary faces $\mathcal{F}^{\mathrm{ext}}$ ), we arrive at

$$
\begin{aligned}
B= & \sum_{i \in N_{S}} B_{i} \\
= & \frac{1}{2} \sum_{s_{i q} \in \mathcal{F}^{\text {in }}} \int_{s_{i q}} \mathbf{J}_{i} \cdot \mathbf{n}_{i q} Q_{q}+\mathbf{J}_{q} \cdot \mathbf{n}_{i q} Q_{i}-\mathbf{J}_{q} \cdot \mathbf{n}_{i q} Q_{i}-\mathbf{J}_{i} \cdot \mathbf{n}_{i q} Q_{q}- \\
& \frac{\alpha}{2} \sum_{s_{i q} \in \mathcal{F}^{\text {int }}} \int_{s_{i q}}\left(\mathbf{J}_{i} \cdot \mathbf{n}_{i q}\left(\mathbf{n}_{i q} \cdot\left(\mathbf{J}_{i}-\mathbf{J}_{q}\right)\right)-\mathbf{J}_{q} \cdot \mathbf{n}_{i q}\left(\mathbf{n}_{i q} \cdot\left(\mathbf{J}_{q}-\mathbf{J}_{i}\right)\right)\right)+ \\
+ & \left.\operatorname{b}^{\text {ext }}\left(Q_{i}-Q_{q}\right)+Q_{q}\left(Q_{q}-Q_{i}\right)\right) \\
= & -\frac{\alpha}{2} \sum_{s_{i q} \in \mathcal{F}^{\text {int }}} \int_{s_{i q}}\left(\left(\mathbf{J}_{i}-\mathbf{J}_{q}\right) \cdot \mathbf{n}_{i q}\right)^{2}+\left(Q_{i}-Q_{q}\right)^{2}+\mathfrak{b}^{\text {ext }} \\
= & -\frac{\alpha}{2}\left(\left\|\mathbf{n} \cdot \llbracket \mathbf{J}_{h} \rrbracket\right\|_{\mathcal{F}^{\text {int }}}^{2}+\left\|\llbracket Q_{h} \rrbracket\right\|_{\mathcal{F} \text { int }}^{2}\right)+\mathfrak{b}^{\text {ext }},
\end{aligned}
$$

and

$$
\begin{aligned}
\mathfrak{b}^{\mathrm{ext}} & =\frac{1}{2} \sum_{s_{i q} \in \mathcal{F}^{\mathrm{ext}}} \int_{s_{i}} \mathbf{J}_{i} \cdot \mathbf{n} Q_{i}-\mathbf{J}_{i} \cdot \mathbf{n} Q_{i}-\frac{\alpha}{2} \sum_{s_{i} \in \mathcal{F}^{\mathrm{ext}}} \int_{s_{i}} \frac{1}{\beta}\left(\left(\mathbf{J}_{i} \cdot \mathbf{n}\right)^{2}+\left(\mathbf{J}_{i} \cdot \mathbf{n}\right)^{2}\right) \\
& =-\alpha\left\|\mathbf{n} \cdot \mathbf{J}_{h}\right\|_{\mathcal{F}^{\text {ext }}}^{2}=0,
\end{aligned}
$$

for the external faces contribution with the appropriate boundary conditions (2.18). The previous results yield

$$
\partial_{t} \mathcal{E}_{h, \mathrm{NL}}(t) \leq 0,
$$

guaranteeing the semi-discrete stability of the nonlocal part. The stability for the generalized model follows readily [24] and is not further detailed here. Consequently, the conclusion follows via classical arguments.

\subsection{Time discretization with a Low Storage Runge Kutta (LSRK) method}

We make use of an explicit LSRK scheme for the time integration. LSRK schemes, compared to standard RK schemes, present the major advantage of requiring only two memory registers per variable, while standard RK algorithms usually require more (more precisely, four registers are necessary for the fourth order standard RK schemes). The LSRK scheme used here is fourthorder accurate, and was specially optimized for an efficient discretization of Maxwell's equations in the DG framework [34]. Since it is not a central point here, the reader is referred to [12] (and references therein) for a more comprehensive introduction to such schemes. Semi-discrete system (3.2) is a system of ODEs in time and can hence be expressed as

$$
\partial_{t} \psi(t)=f(t, \psi(t)) \text {. }
$$

The LSRK scheme, see Algorithm 1, is then applied to (3.33) which yields a fully discrete system in space and time. In [34], Niegemann et al. have proposed schemes with optimized 


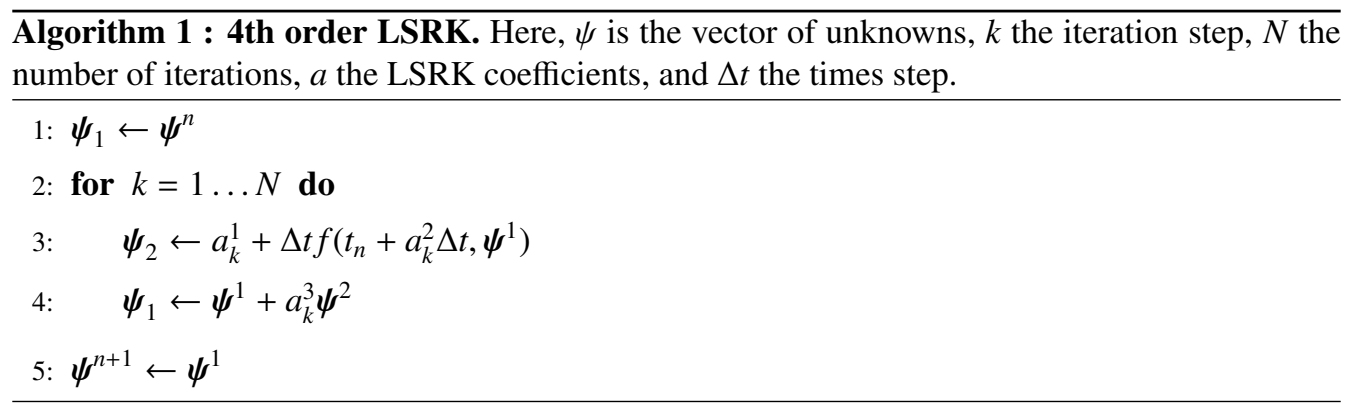

stability regions for the DG-Maxwell operator without dispersion models. However, since the Fermi velocity which enters the linearized fluid model via the $\beta$ parameter is around two orders of magnitude lower than the speed of light, we have never experienced any numerical stability issues with the coupled system (3.2).

\subsection{Curvilinear elements}

Most classical finite element methods rely on tessellations composed of straight-edged elements, regardless of the geometry of the problem. Although this approach presents the advantage of proposing simple transformations of the FE matrices from the reference element to the physical element, in the case of high-order methods as depicted in Figure 2, it represents a serious hindrance, since it limits the accuracy of the spatial discretization to second order. In [33], we proposed an implementation of curvilinear (isoparametric) elements in the DGTD framework for Maxwell's equations, along with local dispersion models for metals. In this case, no additional work was required for local dispersion models, since they only consist of additional ODEs to the Maxwell PDE system. Here, we wish to extend this formulation to non-local dispersion model, in which an additional PDE appears. For the sake of brevity, some basic considerations about the use of curvilinear elements in the DG framework are skipped, and the reader is referred to the aforementioned paper.

The implementation of curvilinear elements in the DG framework requires the numerical integration and storage of the FE matrices on the curvilinear cells only (the method for linear cells remaining the same). As presented in [33], the fully-discrete scheme can be written in terms of (i) the mass matrix $\left(\mathbb{M}_{i}\right)_{j k}=\int_{\Omega_{i}} \phi_{i j} \phi_{i k}$, (ii) the surface matrix $\left(\mathbb{S}_{i l}\right)_{j k}=\frac{1}{2} \int_{\partial \Omega_{i}} \phi_{i j} \phi_{l k}$, and (iii) the stiffness matrices $\left(\mathbb{K}_{i}^{d}\right)_{j k}=\int_{\Omega_{i}} \phi_{i j} \frac{\partial \phi_{i k}}{\partial d}$ for $d \in\{x, y, z\}$. From the numerical integration of such terms, one can easily compute the discrete curl operator on a curvilinear cell, but also the discrete divergence and gradient operators, as they appear in the hydrodynamic equations of formulation (3.2). Hence, no additional numerical integration is required for the computation of volumic matrices when extending the curvilinear DG scheme from the Maxwell equations to the hydrodynamic model. Regarding surfacic terms, it is now necessary to integrate surface integrals of the form $\int_{\partial \Omega_{i}}\left(\mathbf{U}_{i}^{*} \boldsymbol{\phi}_{i k}\right) \cdot \mathbf{n}$ (with its analoguous scalar counterpart) in addition to those appearing 


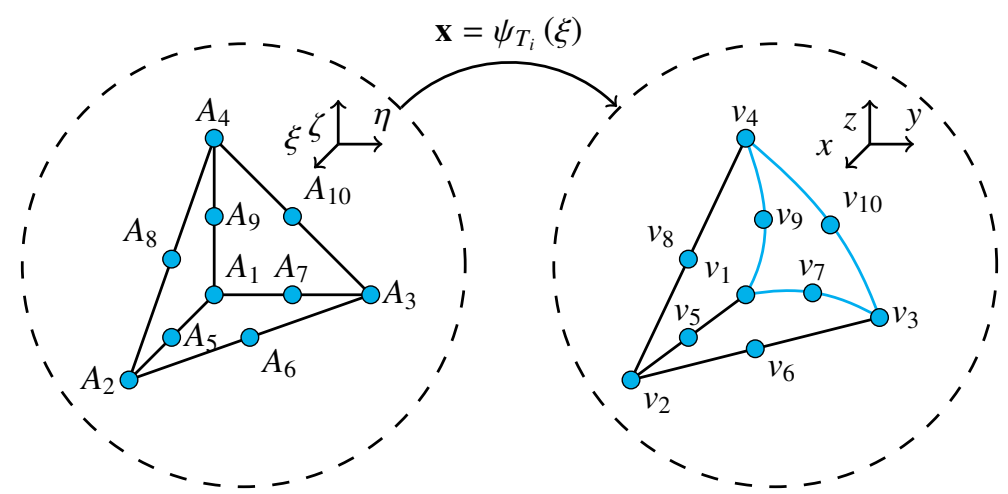

Figure 2: High-order mapping. Second order mapping from the reference element $\hat{T}$ to the physical element $T_{i}$.

in Maxwell's equations $\int_{\partial \Omega_{i}}\left(\mathbf{U}_{i}^{*} \times \boldsymbol{\phi}_{i k}\right) \cdot \mathbf{n}$. As a reminder, it is necessary to develop the integrands of surface integrals including normals terms (since those are not constant over a given curvilinear face), and to compute each integral separately. This work must be done for each curvilinear face, leading to 9 integrals per face for the Maxwell terms, and to 4 additional integrals per face for the hydrodynamic terms. The remaining of the method is identical to what is described in [33], and will not be detailed here.

\section{Numerical results}

This section is concerned with numerical results obtained with our 3D implementation of the proposed DGTD method. We start with two different validation test cases that on the one hand illustrate the numerical convergence rates and on the other hand emphasize the importance of boundary conforming curvilinear elements for scattering problems in nanophotonics. A spherical dimer system eventually concludes our numerical results.

\subsection{Implementation}

System (3.2) has been implemented in the DIOGENeS software suite [35]. Key numerical features, which have not been detailed in this work but that have been used for the following results are

- Total Field/Scattered Field (TFSF) interfaces [36];

- Complex Frequency Shifted Perfectly Matched Layers (CFS-PML) [37];

- Extinction Cross-Section evaluation [19];

- Ohmic loss spectrum evaluation [12];

- Distributed memory (MPI) parallelization.

Moreover, we have used either GMSH [38] or Distene's MeshGems suite [39] for the creation of unstructured tetrahedral affine and curvilinear meshes. 


\subsection{Convergence rates}

In order to validate our implementation of the previously presented DGTD solver we first perform a numerical convergence study by considering a test problem for which we can obtain an analytical time-domain solution. This will be done for (2.16) when neglecting the ODE part. We propose to use the method of manufactured solutions, i.e. we artificially construct a solution that will satisfy (2.15) and hence allows us to determine the convergence rates. Inspired by [40, 21] we exploit the analytical eigenmode solutions of the homogeneous Maxwell's and hydrodynamic equations on a rectangular cavity domain $\Omega_{\square}:=\left\{\mathbf{r} \in\left[0, a_{i}\right]^{3}\right\}$, where $a_{i}>0$ with $i=\{x, y, z\}$ respectively denote the edge lengths in the three cartesian axis. We use PEC boundaries

$$
\mathbf{n}_{\Omega_{\square}} \times\left.\mathbf{E}\right|_{\partial \Omega}=0 \text {, on } \partial \Omega_{\square} \times \mathbb{R}^{+},
$$

for the electromagnetic field and the boundary condition (2.18)

$$
\left.\mathbf{n}_{\Omega_{\square}} \cdot \mathbf{J}\right|_{\partial \Omega}=0 \text {, on } \partial \Omega_{\square} \times \mathbb{R}^{+},
$$

for the hydrodynamic equations, where $\mathbf{n}_{\Omega_{\square}}$ the outward normal to $\Omega_{\square}$. We denote the eigenmode solutions for Maxwell's equations and the hydrodynamic part by $\mathbf{H}^{\text {art }}, \mathbf{E}^{\text {art }}$ and $\mathbf{J}^{\text {art }}, Q^{\text {art }}$, respectively (see [21] for details). We artificially fill this cavity with vacuum for Maxwell's equations and with a dispersive medium characterized by specific values of $\omega_{\mathrm{P}}$ and $\beta$ for the hydrodynamic Drude model while we neglect damping, i.e. $\gamma \equiv 0$. The resulting formulation of the coupled problem including the manufactured solution hence reads

$$
\begin{aligned}
\nabla \times \mathbf{E}+\mu_{0} \partial_{t} \mathbf{H} & =0, \\
\nabla \times \mathbf{H}-\varepsilon_{0} \partial_{t} \mathbf{E} & =\mathbf{J}-\mathbf{J}^{\text {art }}, \\
\beta^{2} \nabla Q-\partial_{t} \mathbf{J} & =-\omega_{\mathrm{P}}^{2} \varepsilon_{0}\left(\mathbf{E}-\mathbf{E}^{\mathrm{art}}\right), \\
\nabla \cdot \mathbf{J}-\partial Q & =0 .
\end{aligned}
$$

Let us define a time dependent error norm $\left\|u-u^{\mathrm{a}}\right\|_{\mathbf{L}^{2}(\Omega)}^{2}$ with $u \in\{\mathbf{H}, \mathbf{E}, \mathbf{J}, Q\}$ and $(\cdot)^{\text {a }}$ being the analytical solution, on the domain $\Omega$ by

$$
e r r=\left\|u-u^{\mathrm{a}}\right\|_{\mathbf{L}^{2}(\Omega)}^{2}:=\sum_{i \in \mathcal{N}_{\Omega}}\left\|u_{i}-u^{\mathrm{a}}\right\|_{\mathbf{L}^{2}\left(\Omega_{i}\right)}^{2}
$$

The convergence rates are computed by comparing the evolution of the error while progressively refining the mesh. Starting with a coarse mesh M1 and successively refining by two (i.e. $h$ becomes $\frac{h}{2}$ at each refinement step) leads to a sixteen times finer mesh M5 (table 2 shows the total number of tetrahedra). Simulations are run for 100 oscillation periods on each mesh, and for each polynomial order. Table 3 summarizes the convergence rates for the DGTD-LSRK scheme with centered fluxes. The results are in line with [34] for the pure Maxwell operator and theoretical results of the DGTD method $[41,18,24]$. We obtain suboptimal convergence rates for the centered scheme, i.e. $n^{\text {th }}$ order for DGTD $-\mathcal{P}_{n}$ with $n$ the polynomial order of the DG ansatz space. Equally, we obtain optimal convergence rates, i.e. $(n+1)^{\text {th }}$ order, for DGTD $-\mathcal{P}_{n}$ if upwind fluxes are used. 
Table 2: Number of tetrahedrons for cubic cavity Mesh M1 is the coarsest and M5 is a systematically refined version of M1.

\begin{tabular}{rrrrrr}
\hline & M1 & M2 & M3 & M4 & M5 \\
\hline \# Tets & 48 & 384 & 3072 & 24576 & 196608 \\
\hline
\end{tabular}

Table 3: Hydrodynamic convergence rates. Numerically obtained convergence rates for the hydrodynamic test cavity solved by the DGTD method with centered fluxes (upper, $\mathcal{P}_{0}$ to $\mathcal{P}_{4}$ ) and upwind fluxes (lower, $\mathcal{P}_{0}$ to $\mathcal{P}_{4}$ ) and a LSRK time integration scheme. Mesh M1 is the most coarsest mesh and M5 the most refined one.

\begin{tabular}{rrrrr}
\hline & $\mathrm{M} 1 \rightarrow \mathrm{M} 2$ & $\mathrm{M} 2 \rightarrow \mathrm{M} 3$ & $\mathrm{M} 3 \rightarrow \mathrm{M} 4$ & $\mathrm{M} 4 \rightarrow \mathrm{M} 5$ \\
\hline $\mathcal{P}_{0}$ & -0.44 & 0.36 & 0.31 & $\mathbf{0 . 0 9}$ \\
$\mathcal{P}_{1}$ & -1.50 & 0.63 & 1.31 & $\mathbf{2 . 0 4}$ \\
$\mathcal{P}_{2}$ & 4.43 & 4.08 & 2.15 & $\mathbf{2 . 4 7}$ \\
$\mathcal{P}_{3}$ & 3.83 & 3.81 & 3.65 & $\mathbf{3 . 0 1}$ \\
$\mathcal{P}_{4}$ & 5.13 & 3.66 & 4.89 & $\mathbf{4 . 1 1}$ \\
& & & & \\
\hline & & & & \\
$\mathcal{P}_{0}$ & 0.03 & 0.08 & 0.11 & $\mathbf{0 . 1 3}$ \\
$\mathcal{P}_{1}$ & 0.12 & 0.77 & 2.29 & $\mathbf{2 . 8 6}$ \\
$\mathcal{P}_{2}$ & 2.59 & 4.48 & 3.69 & $\mathbf{3 . 0 6}$ \\
$\mathcal{P}_{3}$ & 5.68 & 4.19 & 3.96 & $\mathbf{4 . 0 1}$ \\
$\mathcal{P}_{4}$ & 4.68 & 4.24 & 4.08 & $\mathbf{4 . 0 7}$ \\
\hline
\end{tabular}




\subsection{Mie scattering of a single sphere}

The scattering of a single sphere in vacuum is a classic nanophotonic benchmark example. Beyond applications where the relatively simple result of an isolated sphere in vacuum already explains several physical effects, the scattering of a single sphere problem also comes with an analytical solution. This, of course, permits us to compare the performance of our method with so called Mie solutions. Gustav Mie has published his work on the scattering of spheres in vacuum in the early $20^{\text {th }}$ century [42] and his initial works have been extended to more complex material configurations [43], different coordinate systems [44] and even nonlocal dispersion laws $[45,30,46]$.

From a more methodological point of view, the Mie sphere appears to be significantly more complex in comparison to the test problem of a cubic cavity that we have used for the convergence study in the previous section. Among others, the sphere involves the additional boundary condition (2.17) at the interface between the metallic sphere itself and the surrounding vacuum. Furthermore, it demands a free space approximation of the infinite vacuum. Regarding the free space approximations, we have used CSF-PMLs [47, 37] in order to minimize spurious reflections from the artificial domain truncation. Since such a setup forbids a direct imposition of the incident field, i.e. a pulsed plane wave in our case, via for example first-order Silver-Müller radiation conditions [16], we employ a TF/SF approach [36].

We now investigate the surface approximation of a single sphere and its influence on the resulting extinction Cross-Section (CS) spectra. Here, we compare different polynomial orders of the DG basis functions as well as the accuracy, and hence performance and gain if curvilinear tetrahedra are used. Figure 3 shows the computational meshes we have used for this study. There are two topologically different meshes M1 and M2, shown in Figure 3 (a) and (c), respectively. Both meshes represent the same physical sphere and the geometrical misrepresentation of M1 (a) is quite obvious as the sphere hardly resembles a sphere at all - it rather represents a faceted particle. Performing an $h$-refinement leads to M2 (c) which visibly better approximates a sphere. Activating curvilinear elements gives curved versions of M1 and M2 as depicted in Figure 3 (b) and (d).

The incident pulsed plane wave that is injected by the TF/SF interface follows a Gaussian modulated harmonic time signal of the temporal form

$$
\left\{\mathbf{E}_{\mathrm{inc}}, \mathbf{H}_{\mathrm{inc}}\right\} \propto \sin \left(\omega_{\mathrm{c}}(t-\tau)\right)\left(-\left(\frac{t-\tau}{\sigma}\right)^{2}\right) .
$$

Here, $\mathbf{E}_{\mathrm{inc}}, \mathbf{H}_{\mathrm{inc}}, \omega_{\mathrm{c}}, \tau$ and $\sigma$ respectively are the incident electric and magnetic field strength, the centered angular frequency, a time shift and the broadening of the pulse (depending on the considered frequency range). The spatial profile is equivalent to a monochromatic plane wave [22] and depends on the incident direction, i.e. the polarization of the wave. In the sphere case, the polarization direction does not matter due to the radial symmetry.

We have chosen the extinction CS as the measured Quantity of Interest (QoI) defined by

$$
C_{\text {ext }}:=C_{\mathrm{abs}}+C_{\mathrm{sca}},
$$

and refer to $[19,12]$ for further details of the definitions of the absorption and scattering CS. The $\mathrm{CS}$ is evaluated on the same TF/SF interface on which the incident field is injected.

Having set up the simulation framework including the source, the boundary condition and the QoI, we can discuss the obtained results as outlined in Figures 4 and 5 for the two meshes, respectively. Obviously, mesh M1 with affine tetrahedra leads to a very poor result, similarly 
for first order $\mathcal{P}_{1}$ and fourth $\operatorname{order} \mathcal{P}_{4}$ polynomials as shown in Figure 4. The $\mathcal{P}_{1}$ solution only reproduces the first resonance at about $0.675 \omega_{\mathrm{P}}$ although it is supposed to be at $0.7 \omega_{\mathrm{P}}$. Additionally, the second and third resonance of the Mie spectrum at about $0.825 \omega_{\mathrm{P}}$ and $0.875 \omega_{\mathrm{P}}$ are completely inobservable. Increasing the polynomial order to $\mathcal{P}_{4}$ slightly improves the situation and the second resonance seems to build up. However, both resonances positions are far off and the absolute as well as the relative amplitudes completely fail with respect to the Mie solution. If we now switch to the curvilinear version of M1, the situation drastically changes. The $\mathcal{P}_{1}$ solution suddenly shifts the first resonance almost to the correct angular frequency, the second resonance now appears in the spectrum and even relatively close to the correct resonance angular frequency. A third resonance seems to build up at $0.95 \omega_{\mathrm{P}}$ which would be quite far from the correct position if we relate it to the actual third resonance in the Mie spectrum. Again, increasing the polynomial order to $\mathcal{P}_{4}$ the numerical solution significantly approaches the analytical Mie solution down to an error of $2.38 \%$. Table 4 summarizes the errors of all runs.

Spatial $h$-refinement refinement of the sphere, i.e. the mesh M2, improves the situation of the linear solutions. Figure 5 depicts the $\mathcal{P}_{1}$ spectrum now showing the first resonance at the correct frequency and the second resonance that now builds up at $0.815 \omega_{\mathrm{P}}$ instead $0.825 \omega_{\mathrm{P}}$. Although the positions of the resonances are relatively close to the exact solution, the amplitudes still differ heavily. The third resonance remains invisible for first order polynomials. If fourth order polynomials $\mathcal{P}_{4}$ on linear elements are used, the numerical solution already gets fairly close to the exact solution with an error of 5.45\%. Activating curvilinear elements for $\mathcal{P}_{1}$ and $\mathcal{P}_{4}$, the numerical solutions with respect to the linear runs increase equally to M1 and the obtained error for $\mathcal{P}_{4}$ only is $0.96 \%$.

Curvilinear simulations are intrinsically more costly than linear ones due to increased memory consumption, a reduced CFL condition and additional DoFs, see section 3.6 for details, in comparison to the same run on a linear mesh. In order to present a fair comparison of a curvilinear solution on the coarse mesh M1 and the solution on an $h$-refined mesh M2 with linear elements, we choose the two runs with a similar error level, namely M1 $-\mathcal{P}_{3}-$ curv and M $2-\mathcal{P}_{4}-$ linear. Figure 6 depicts the obtained spectra and Table 5 lists the statistics of both simulations and sequential as well as parallel.

Both meshes almost have the same amount of cells since the largest part of the domain consists of vacuum. While the sphere is discretized by 46 curved tetrahedra for M1, the $h$-refined mesh M2 needs 292 elements. This local refinement explains the little relative difference in the total amount of mesh cells and is at the same time close to a real world nanophotonic. Of course, the finer mesh M2 suffers from more mesh cells and even more severely from a reduced CFL condition due to a smaller characteristic mesh length $h$ and an increased polynomial degree [18], which increases the needed number of iterations in the time stepping scheme from 7060 for M1 to 90933 for M2. An average runtime for 100 time stepping iterations of $374 \mathrm{~s}$ and $703 \mathrm{~s}$ for M1 and M2, respectively leads to a total simulation time of $7 \mathrm{~h} 20 \mathrm{mn}$ and $177 \mathrm{~h} 34 \mathrm{mn}$ which means a performance speed-up of 24.2. In terms of memory, the M1 $-\mathcal{P}_{3}$ - curv solution comes with more DoFs in comparison with a linear M1 run but still better performs in comparison to a $\mathrm{M} 2-\mathcal{P}_{4}-$ lin run by $75 \%$.

If MPI parallelization is used (only within a single multicore node in this example) we obtain reasonably good scaling results. As depicted in Table 5 the scaling slightly breaks down at 12 subdomains for M1 and already at 8 subdomains for M2. This is due to the quite inhomogeneous load balancing of the computational cost of each cell type. The performed simulations contain different types of equations to be solved on a cell: purely Maxwell's equations in vacuum, Maxwell plus the hydrodynamic equation in the sphere, CFS-PML cells, and boundary 


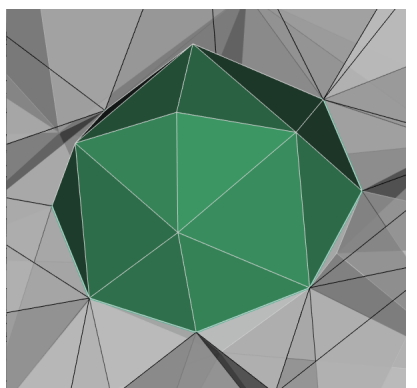

(a) Mesh M1 without high-order cells.

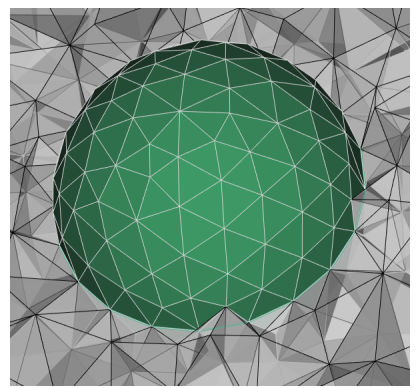

(c) Mesh M2 without high-order cells.

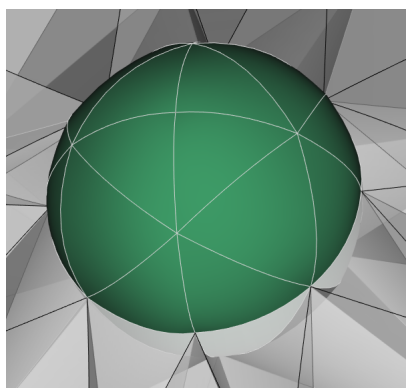

(b) Mesh M1 with high-order cells.

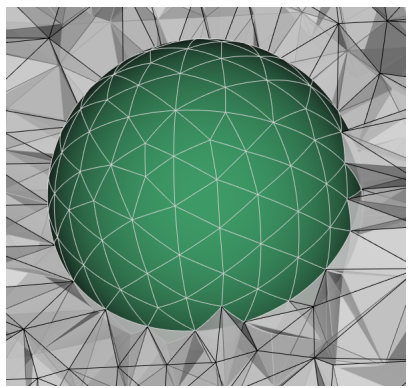

(d) Mesh M2 with high-order cells.

Figure 3: Four different discretizations for a scattered sphere in vacuum. Figure (a) and (b) have the same number of elements and differ by the geometric representation order from linear to quadratic. Figure (c) is a refined version of (a) and (d) the high-order version.

cells. Additionally, the TF/SF incident field and on-the-fly Fourier transform of the extinction CS will add to an even stronger imbalance. The systematic optimization of the load balancing will be part of a future work.

\subsection{Impact of nonlocal dispersion on spherical dimers}

Spherical dimer systems consist of two spheres being very close to each other, hence forming a gap system, see Figure 7a. Such dimer gap systems are well known to show high field enhancements due to the hybridized modes of the individual spheres that build up a so called gap plasmon. These field enhancements are of interest for e.g. Surface Enhanced Raman Scattering (SERS) [48].

Beyond the applicative aspects, the spherical dimer system is significantly more complicated for analytical solutions due to a lower symmetry level than single spheres. Breaking the symmetry of a single sphere by introducing a second sphere brings a second tuning parameter of the system - the gap size. For sufficiently small gaps, nonlocal effects are expected to occur, as has been recently theoretically shown for a fully retarded three dimensional dimer case [49].

For extinction CS simulations, in contrast to scattering CS, the absorption CS comes into play and the near field thus gains importance. A precise evaluation of the absorption CS, in turn, requires a well resolved near field which strongly depends on the surface approximation of the nano particles. 


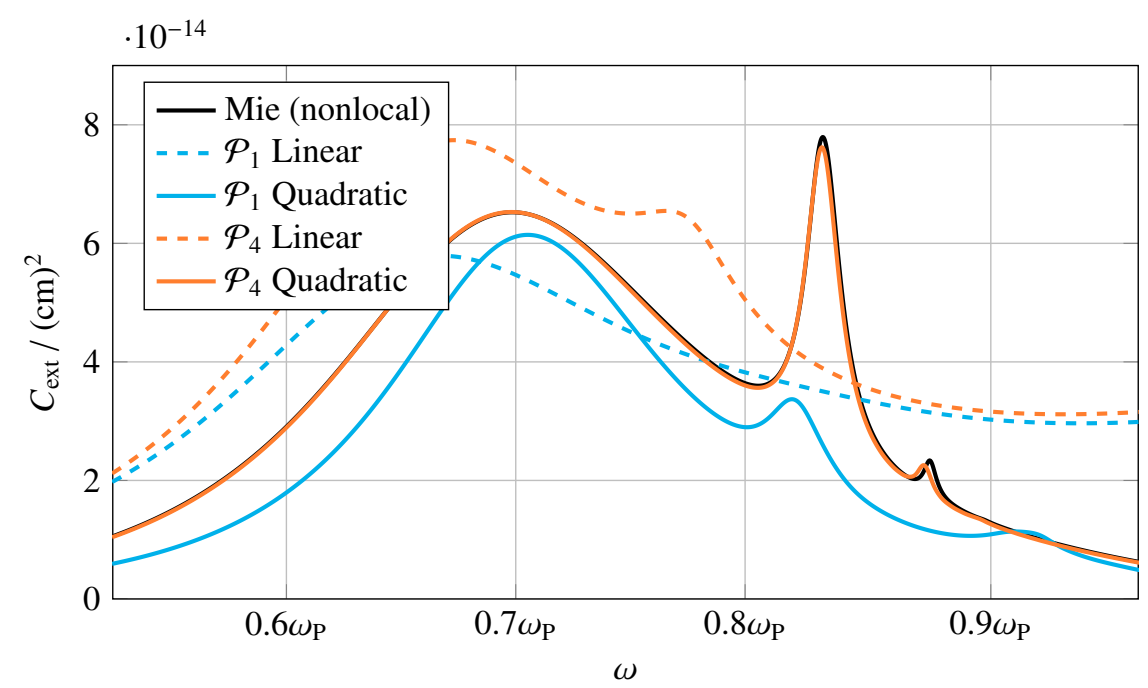

Figure 4: Extinction cross-section spectra on mesh M1. Comparison of the extinction cross-section spectra of a metallic nanosphere in dependence of the polynomial interpolation order $\mathcal{P}_{n}$ and the geometric mesh order, i.e. linear or quadratic mesh elements. The error in comparison to the Mie solution can be found in Table 4

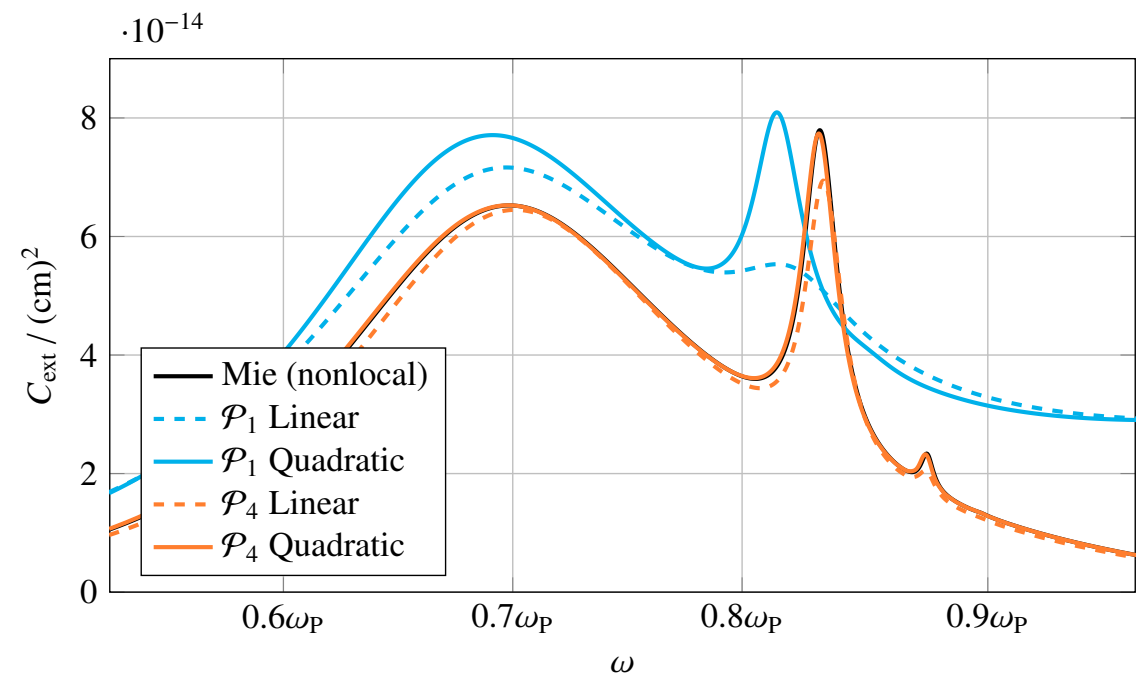

Figure 5: Extinction cross-section spectra on mesh M2. Comparison of the extinction cross-section spectra of a metallic nanosphere in dependence of the polynomial interpolation order $\mathcal{P}_{n}$ and the geometric mesh order, i.e. linear or quadratic mesh elements. The error in comparison to the Mie solution can be found in Table 4 


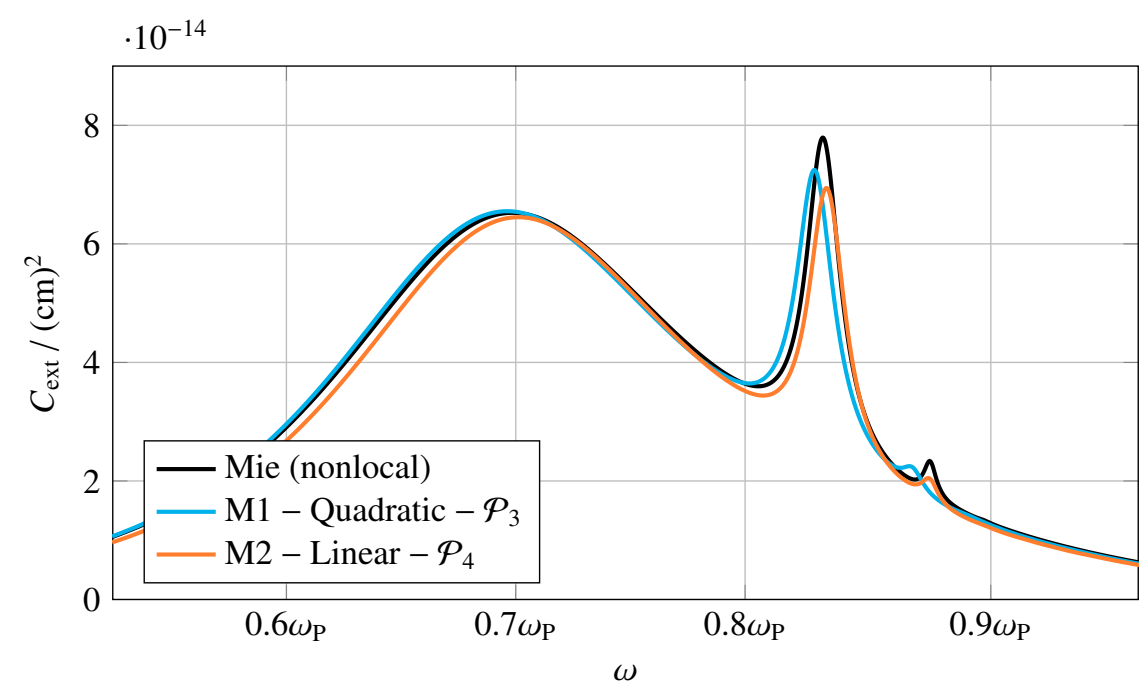

Figure 6: Extinction cross-section spectra on mesh M1. Comparison of the extinction cross-section spectra of a metallic nanosphere in dependence of the polynomial interpolation order $\mathcal{P}_{n}$ and the geometric mesh order, i.e. linear or quadratic mesh elements. The error in comparison to the Mie solution can be found in Table 4

Table 4: Relative error of sphere simulations. The relative error is given with respect to the analytical nonlocal Mie solution.

\begin{tabular}{rrrrr}
\hline & $\mathcal{P}_{1}$ & $\mathcal{P}_{2}$ & $\mathcal{P}_{3}$ & $\mathcal{P}_{4}$ \\
\hline M1 - Linear & $33.33 \%$ & $29.95 \%$ & $28.83 \%$ & $28.80 \%$ \\
M1 - Quadratic & $43.67 \%$ & $18.51 \%$ & $6.60 \%$ & $2.38 \%$ \\
M2 - Linear & $33.50 \%$ & $11.07 \%$ & $7.18 \%$ & $5.34 \%$ \\
M2 - Quadratic & $39.40 \%$ & $10.07 \%$ & $3.58 \%$ & $0.96 \%$ \\
\hline
\end{tabular}


Table 5: Performance comparison of Sphere simulations. Sequential mesh and CPU statistics of the mesh M1 with curvilinear elements and a polynomial order $\mathcal{P}_{3}$ versus the refined mesh M2 with rectilinear elements and a polynomial $\operatorname{order} \mathcal{P}_{4}$. The simulated spectra are depicted in Figure 6 . The listed values only contain the actual mesh and do not contain ghost cells due to boundary conditions and domain decomposition for the parallel MPI runs. These runs have been performed on an Intel $($ Xeon $(C P U$ E5-2630 v2 $2.6 \mathrm{GHz}$ with 64 GB RAM machine.

\begin{tabular}{|c|c|c|c|c|c|c|c|c|}
\hline & \multicolumn{6}{|c|}{ M1 - Quadratic $-\mathcal{P}_{3}$} & \multicolumn{2}{|c|}{ M2 - Linear $-\mathcal{P}_{4}$} \\
\hline L2-error (Mie) & & & & $6.6 \%$ & & & & $5.34 \%$ \\
\hline \# Cells & & & & 13827 & & & & 14334 \\
\hline \# Sphere cells & & & & 46 & & & & 292 \\
\hline \# HO cells & & & & 200 & & & & - \\
\hline \# Iterations & & & & 7060 & & & & 90933 \\
\hline Memory & & & & $481.5 \mathrm{MB}$ & & & & $1980.4 \mathrm{MB}$ \\
\hline \multicolumn{9}{|l|}{ Time per } \\
\hline 100 iterations & & & & $374 \mathrm{~s}$ & & & & $703 \mathrm{~s}$ \\
\hline \multicolumn{9}{|l|}{ Total time in } \\
\hline loop (sequ.) & & & & $26404 \mathrm{~s}$ & & & & $639259 \mathrm{~s}$ \\
\hline \multirow[t]{2}{*}{ Speed-up } & & & & 24.2 & & & & - \\
\hline & \#proc & $T[\mathrm{~s}]$ & $T_{\mathrm{cpu}}[\mathrm{s}]$ & speed-up & \#proc & $T[\mathrm{~s}]$ & $T_{\mathrm{cpu}}[\mathrm{s}]$ & speed-up \\
\hline \multirow{4}{*}{$\begin{array}{l}\text { Timer per } 100 \\
\text { iterations (parallel) }\end{array}$} & 2 & 186 & 372 & 2.0 & 2 & 380 & 740 & 1.9 \\
\hline & 4 & 94 & 376 & 4.0 & 4 & 200 & 800 & 3.5 \\
\hline & 8 & 50 & 400 & 7.5 & 8 & 105 & 840 & 6.7 \\
\hline & 12 & 37 & 444 & 10.1 & 12 & 73 & 876 & 9.6 \\
\hline
\end{tabular}




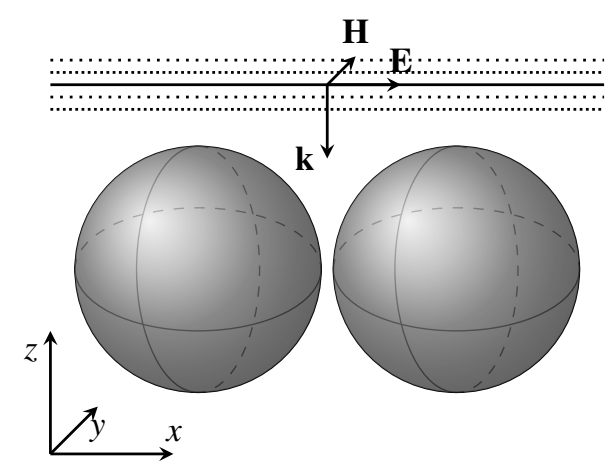

(a) Spherical dimer system. The spherical dimer system consists of two equivalent spheres with a diameter $d=20 \mathrm{~nm}$ and a gap size at the closest points of $\delta=$ $2 \mathrm{~nm}$. The incoming plane wave's electric field is polarized along the dimer axis $\mathbf{e}_{x}$ and the physical parameters are $\omega_{\mathrm{P}}=1.39 \cdot 10^{16} \mathrm{rad} / \mathrm{s}, \gamma=3.23 \cdot 10^{13} \mathrm{rad} / \mathrm{s}$, $\beta=0.84 \cdot 10^{6} \mathrm{~m} / \mathrm{s}$.

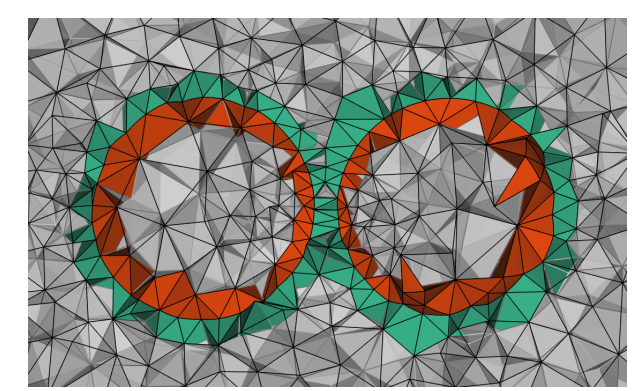

(b) Computational dimer mesh. Red: curvilinear elements inside the sphere, green: curvilinear elements in the surrounding vacuum. All transparent gray elements are linear and consist of either vacuum if outside the red elements or metal if inside.

Figure 7: Spherical dimer setup. Figure (a) shows the principle setup and (b) the computational mesh.

Figure 8 shows the extinction CS spectra for two simulations on a mesh with affine tetrahedra and a converged reference spectrum obtained on a curvilinear mesh. The solution with centered fluxes, i.e. $\alpha=0$ in (3.8), barely shows dissipation and almost contains all the resonances as the reference solution. Unfortunately, the spectrum is heavily polluted by spurious oscillations which makes it extremely difficult for the weaker resonances of amplitudes below $3 \cdot 10^{-15} \mathrm{~cm}^{-2}$ to be correctly distinguished and detected. Switching to upwind fluxes, i.e. $\alpha=1.0$ in (3.8), damps away all spurious solutions at the price of higher numerical dissipation making the detection of weak resonances impossible again.

If curvilinear elements are turned on now, i.e. the black curve in Figure 8, we indeed see that the previously discussed centered spectrum almost contains all correct resonances plus additional spurious modes while the upwind solution smoothes out too strongly and at least two resonances are completely lost. Figure $7 \mathrm{~b}$ illustrated the computational mesh of the dimer system with the curvilinear elements being colored in red for metal and green for vacuum.

Comparing a local dispersion model $\beta=0$ with the nonlocal solution in Figure 9 shows significant blue-shifts for all resonances. The resonances above $0.6 \omega_{\mathrm{P}}$ are even shifted strong enough to lie on the next local resonance.

\subsection{Nonlocal sensitive gap plasmon for nano cubes}

Our last simulation example is concerned with the demonstration of a significant influence of the nonlocal dispersion model on the gap plasmon resonance in a nano cube setup. Nano cubes are particularly interesting in the present modeling context because of the high mode confinement in the gap between the cube and the metallic ground plate which is known to be highly sensitive to nonlocality in comparison to e.g. a standard surface plasmon [14].

Realistic simulations of such nano cubes face several challenges from a numerical point of view especially due to the necessary edge and corner roundings [12], which cause severe difficulties for modal methods and cartesian grid based algorithms. Even for methods based on unstructured methods, these roundings stay challenging as they are relatively small compared 


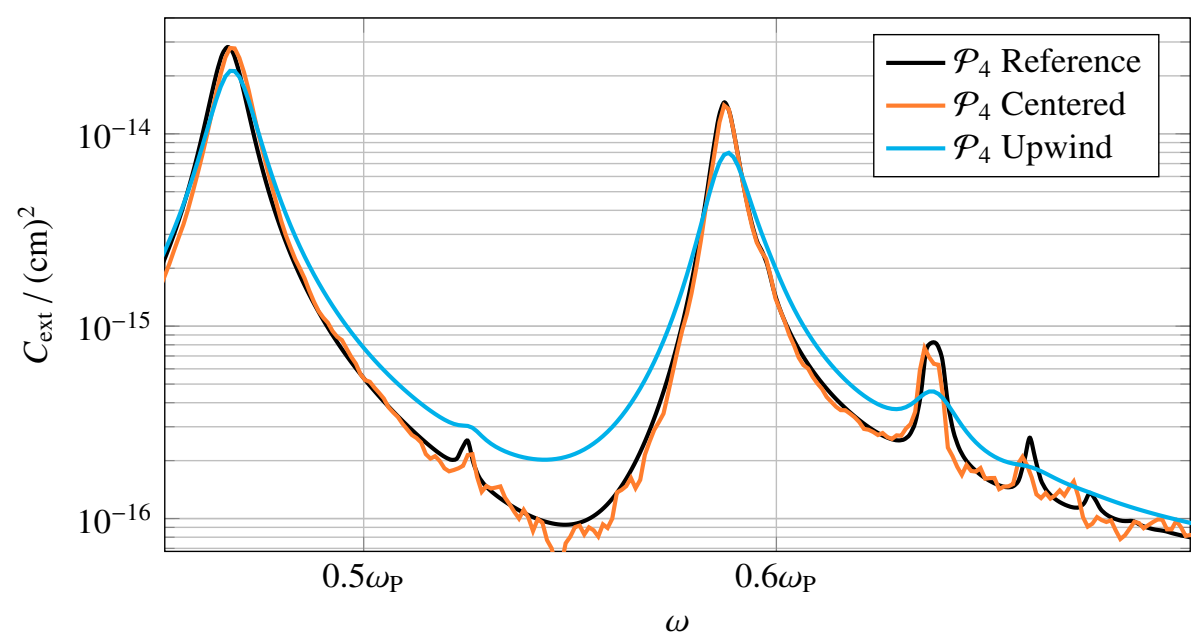

Figure 8: Extinction cross-section spectra of the spherical dimer. Comparison of the extinction cross-section spectra for a spherical dimer system on a linear mesh in dependence of the numerical flux choice. Black: the reference DGTD solution with quadratic elements and upwind fluxes. Orange: DGTD with centered fluxes and linear mesh cells, and blue: DGTD with upwind fluxes and linear mesh cells.

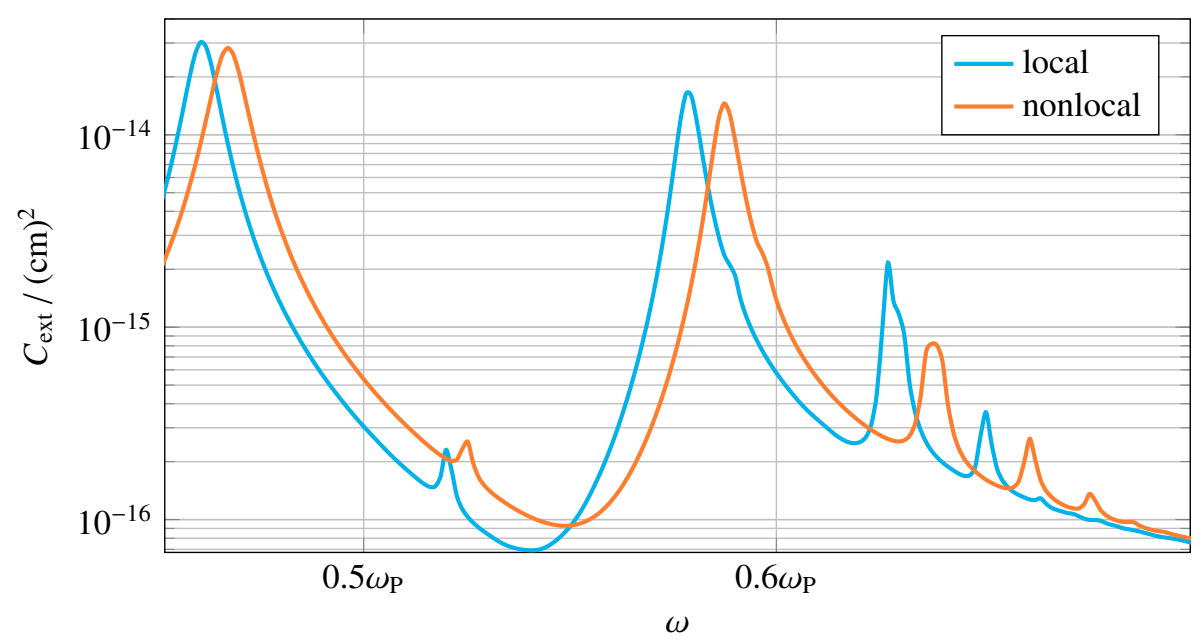

Figure 9: Extinction cross-section spectra of the spherical dimer. Comparison of the extinction cross-section spectra for a spherical dimer system in dependence of the material model. 
to the cube length and would hence demand an extreme local refinement in the vicinity of the edges and corners. This leads to a higher amount of mesh cells and further restricts the time step condition of the explicit time integration scheme. Employing curvilinear elements overcomes this restriction as shown in Figure 10a.

Beyond purely numerical challenges, the nano cube setup requires the consideration of bound and free electrons. For this reason, we employ the model we have proposed and studied in section 2.5 which takes into account bound electrons, as well as free electrons by means of a nonlocal Drude model.

Figure 10b depicts the simulated loss spectra. While the first peak is not linked to gap plasmon and does only show a very weak blue-shift of $\Delta \lambda \approx 4 \mathrm{~nm}$, the second resonance is due to the gap mode and obviously more sensitive leading to a blue-shift of $\Delta \lambda \approx 35 \mathrm{~nm}$. Additionally, the local dispersion model seems to overestimate ohmic losses with respect to the nonlocal model.

More comprehensive studies of this setup including more geometric details of the complete setup and the comparison to experimental data are part of a future work.

\section{Conclusion and Outlook}

In this work, we have set up the modelling of bound and free electrons for spatially dispersive metal structures in nanophotonics. We have proposed to use a generalized dispersion model for the bound electron contribution in combination with a linearized hydrodynamic electron model for the spatially dispersive free electrons of the metal.

Furthermore, we have applied a Discontinuous Galerkin Time Domain discretization to the complete system, consisting of Maxwell's equations, a generalized dispersion model and the nonlocal hydrodynamic equations. We have provided the corresponding upwind fluxes for the complete system and have studied the semi-discrete stability of the scheme.

The proposed DGTD implementation is high order in space and time and is ready to run on modern High Performance Computing clusters. We have obtained important performance gains thanks to the discretization of curved material interfaces with quadratic tetrahedra. Eventually, we have demonstrated a significant influence of spatial dispersion on spherical dimer systems and on single nanocubes.

In future works, we will continue to investigate more physically challenging test cases, and will also focus on improving our material models by including the effect of electron spill-out.

Acknowledgment. Many thanks to Dr. Antoine Moreau, Dr. Matthias Moeferdt and Prof. Kurt Busch for the fruitful discussions about the results of this work. 


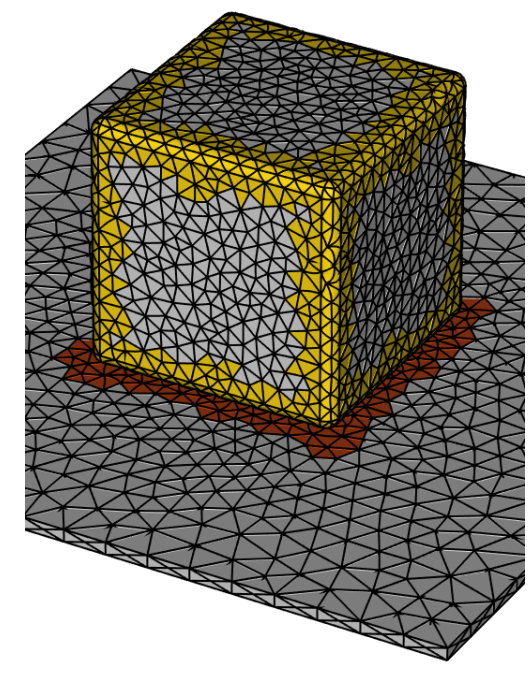

(a) Nano cube mesh. The nano cube setup consists of a metallic ground plate, a dielectric spacer (red) and the metallic cube itself (yellow). Here, only the dielectric spacer and the cube are displayed where the linear tetrahedral are gray, independent of the domain and the curvilinear elements are colored. Red: dielectric, yellow: metal. The dielectric spacer measures $\delta=3 \mathrm{~nm}$, the cube length $L_{\text {cube }}=$ $60 \mathrm{~nm}$ and the radius of the edges and corners $r_{\text {cube }}=3 \mathrm{~nm}$.

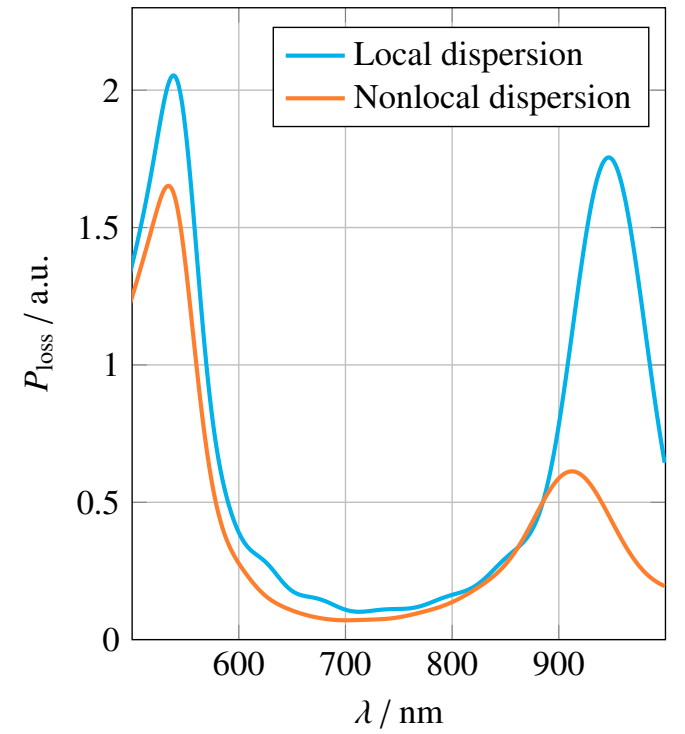

(b) Loss spectrum. Loss spectra of the nano cube with a locally dispersive gold versus nonlocally dispersive gold. The exact resonance positions for the local run are $\lambda_{\text {res, local }}=$ $\{539,947\} \mathrm{nm}$ and $\lambda_{\text {res, nonlocal }}=\{534,912\} \mathrm{nm}$, respectively.

Figure 10: Nano cube mesh and result. Figure (a) shows the curvilinear tetrahedral mesh of and (b) simulated loss spectrum. The gold in the ground plane as well as the cube consist of gold with the parameters from Table 1 for the bound contribution and $\omega_{\mathrm{P}}=1.195993 \cdot 10^{16} \mathrm{rad} / \mathrm{s}, 8.052118 \mathrm{rad} / \mathrm{s}$ and $\beta=1.35 \mathrm{~m} / \mathrm{s}$ for the Drude part. The dielectric of the spacer is not dispersive in this frequency regime and we have used $\varepsilon_{\mathrm{r}}=2.3104$. 
[1] S. Gaponenko, Introduction to nanophotonics, Cambridge University Press, 2010.

[2] R. Bakker, Y. Yu, R. Paniagua-Domnguez, B. Luk'yanchuk, A. Kuznetsov, Resonant light guiding along a chain of silicon nanoparticles, Nano Lett. 17 (2017) 3458-3464.

[3] P. Genevet, F. Capasso, Holographic optical metasurfaces a review of current progress, Rep. Progr. Phys. 78 (2015) 024401.

[4] N. Mauranyapin, L. Madsen, M. Taylor, M. Waleed, W. Bowen, Evanescent single-molecule biosensing with quantum-limited precision, Nat. Photon. 11 (2017) 477-481.

[5] G. Sotiriou, F. Starsich, A. Dasargyri, M. Wurnig, F. Krumeich, A. Boss, J.-C. Leroux, S. Pratsinis, Photothermal killing of cancer cells by the controlled plasmonic coupling of silica-coated $\mathrm{Au} / \mathrm{Fe} 2 \mathrm{O} 3$ nanoaggregates, Adv. Funct. Mater. 24 (2014) 2818-2827.

[6] S. Maier, Plasmonics - Fundamentals and applications, Springer, 2007.

[7] F. Chen, Introduction to plasma physics and controlled fusion: plasma physics, Springer, 3rd edition, 2016.

[8] P. Drude, Zur elektronentheorie der metalle, Annalen der Physik 306 (1900) 566-613.

[9] P. Johnson, R. Christy, Optical constants of the noble metals, Phys. Rev. B 6 (1972) 4370-4379.

[10] F. Hao, P. Nordlander, Efficient dielectric function for FDTD simulation of the optical properties of silver and gold nanoparticles, Chem. Phys. Lett. 446 (2007) 115-118.

[11] A. Rakic, A. Djuris, J. Elazar, M. Majewski, Optical properties of metallic films for vertical-cavity optoelectronic devices, Appl. Opt. 37 (1998) 5271-5283.

[12] J. Viquerat, Simulation of electromagnetic waves propagation in nano-optics with a high-order discontinuous Galerkin time-domain method, Ph.D. thesis, University Nice Sophia Antipolis, 2016.

[13] A. Moreau, C. Ciracì, D. Smith, The impact of nonlocal response on metallo-dielectric multilayers and optical patch antennas, Phys. Rev. B (2012) 1-12.

[14] A. Pitelet, E. Mallet, E. Centeno, A. Moreau, Fresnel coefficients and Fabry-Perot formula for spatially dispersive metallic layers, Phys. Rev. B 96 (2017) 1-5.

[15] K. Savage, M. Hawkeye, R. Esteban, A. Borisov, J. Aizpurua, J. Baumberg, Revealing the quantum regime in tunnelling plasmonics, Nature 491 (2012) 574-577.

[16] P. Monk, Finite element methods for Maxwell's equations, Oxford Science Publications, 2003.

[17] J. Nedelec, A new family of mixed finite elements in $\mathbb{R}^{3}$, Numer. Math. 50 (1986) 57-81.

[18] J. Hesthaven, T. Wartburton, Nodal Discontinuous Galerkin methods, Springer, 2008.

[19] K. Busch, M. König, J. Niegemann, Discontinuous Galerkin methods in nanophotonics, Laser and Photonics Reviews 5 (2011) 1-37.

[20] J. Niegemann, Higher-order methods for solving Maxwell's equations in the time-domain, Ph.D. thesis, Fakultät für Physik des Karlsruher Instituts für Technologie, 2009.

[21] N. Schmitt, C. Scheid, S. Lanteri, A. Moreau, J. Viquerat, A DGTD method for the numerical modeling of the interaction of light with nanometer scale metallic structures taking into account non-local dispersion effects, J. Comput. Phys. 316 (2016) 396-415.

[22] J. D. Jackson, Classical electrodynamics, John Wiley \& Sons, 3rd edition, 1998.

[23] C. Kittel, Introduction to solid state physics, Wiley, 2004.

[24] S. Lanteri, C. Scheid, J. Viquerat, Analysis of a generalized dispersive model coupled to a DGTD method with application to nanophotonics, SIAM J. Sci. Comp. 39 (2017) 831-859.

[25] A. Boardman, Electromagnetic surface modes, John Wiley \& Sons, 1982.

[26] C. Ciracì, F. Della Sala, Quantum hydrodynamic theory for plasmonics: impact of the electron density tail, Phys. Rev. B 93 (2016) 205405.

[27] C. Ciracì, R. Hill, J. Mock, Y. Urzhumov, A. Fernández-Domínguez, S. Maier, J. Pendry, A. Chilkoti, D. Smith, Probing the ultimate limits of plasmonic enhancement, Science 337 (2012) 1072-1074.

[28] M. Kauranen, A. Zayats, Nonlinear plasmonics, Nat. Photon. 6 (2012) 737-748.

[29] S. Raza, Probing plasmonic nanostructures with electron energy-loss spectroscopy, Ph.D. thesis, DTU Fotonik, Technical University of Denmark, 2014.

[30] T. Christensen, W. Yan, S. Raza, A. Jauho, N. Mortensen, M. Wubs, Nonlocal response of metallic nanospheres probed by light, electrons, and atoms, ACS Nano 8 (2014) 1745-1758.

[31] K. Hiremath, L. Zschiedrich, F. Schmidt, Numerical solution of nonlocal hydrodynamic Drude model for arbritrary shaped nano-plasmonic structures using Nedelec finite elements, J. Comput. Phys. 231 (2012) 5890-5896.

[32] C.-W. Shu, An analysis of three different formulations of the Discontinuous Galerkin method for diffusion equations, Math. Mod. Meth. Appl. Sci. 13 (2003) 395-413.

[33] J. Viquerat, C. Scheid, A 3D curvilinear discontinuous Galerkin time-domain solver for nanoscale light-matter interactions, J. Comput. Appl. Math. 289 (2015) 37-50.

[34] J. Niegemann, R. Diehl, K. Busch, Efficient low-storage Runge-Kutta schemes with optimized stability regions, J. Comput. Phys. 231 (2012) 364-372.

[35] J. Viquerat, N. Schmitt, A. Gobé, S. Lanteri, C. Scheid, DIOGENeS: A DG-based software suite for nano-optics, 
2017.

[36] A. Taflove, S. Hagness, Computational electrodynamics: the finite-difference time-domain method, Artech House Publishers, 3rd edition, 2005.

[37] M. König, C. Prohm, K. Busch, J. Niegemann, Stretched-coordinate PMLs for Maxwell's equations in the discontinuous Galerkin time-domain method, Opt. Expr. 19 (2011) 4618-4631.

[38] C. Geuzaine, GMSH: a three-dimensional finite element mesh generator with built-in pre- and post-processing facilities, Int. J. Numer. Meth. Engrg. 79 (2009) 1309-1331.

[39] MeshGems: a suite of reliable meshing software components for high-demanding CAD/CAE developers, 2016.

[40] S. Lanteri, C. Scheid, Convergence of a discontinuous Galerkin scheme for the mixed time domain Maxwell's equations in dispersive media, IMA J. Numer. Anal. 33 (2013) 432-459.

[41] L. Fezoui, S. Lanteri, S. Lohrengel, S. Piperno, Convergence and stability of a discontinuous Galerkin time-domain method for the 3D heterogeneous Maxwell equations on unstructured meshes, ESAIM: Math. Model. Numer. Anal. 39 (2005) 1149-1176.

[42] G. Mie, Beitraege zur optik trüber medien, speziell kolloidaler metalllösungen, 1908.

[43] D. Cohoon, An exact solution of Mie type for scattering by a multilayer anisotropic sphere, J. Electromag. Wav. Applic. 3 (1989) 421-448.

[44] M. Moeferdt, Nonlocal and nonlinear properties of plasmonic nanostructures within the hydrodynamic Drude model, Ph.D. thesis, Mathematisch-Naturwissenschaftliche Fakultät, Humboldt-Universität zu Berlin,, 2017.

[45] R. Ruppin, Optical properties of a plasma sphere, Phys. Rev. Lett. 31 (1973) 1434-1437.

[46] C. David, F. García De Abajo, Spatial nonlocality in the optical response of metal nanoparticles, J. Phys. Chem. C 115 (2011) 19470-19475.

[47] J.-P. Berenger, Perfectly Matched Layer (PML) for computational electromagnetics, Morgan \& Claypool All, 2007.

[48] H. Xu, E. Bjerneld, M. Kall, L. Borjesson, Spectroscopy of single hemoglobin molecules by surface enhanced Raman scattering, Phys. Rev. Lett. 83 (1999) 4357.

[49] N. Schmitt, J. Viquerat, C. Scheid, S. Lanteri, M. Moeferdt, K. Busch, A 3D discontinuous Galerkin time-domain method for nanoplasmonics with a nonlocal dispersion model, in: Progress In Electromagnetics Research Symposium - PIERS 2017, St Petersburg, Russia. 